\title{
Stability of Regime-Switching Diffusion Systems with Discrete States Belonging to a Countable Set*
}

\author{
Dang Hai Nguyen ${ }^{\dagger} \quad$ George Yin ${ }^{\ddagger}$
}

October 10, 2017

\begin{abstract}
This work focuses on stability of regime-switching diffusions consisting of continuous and discrete components, in which the discrete component switches in a countably infinite set and its switching rates at current time depend on the continuous component. In contrast to the existing approach, this work provides more practically viable approach with more feasible conditions for stability. A classical approach for asymptotic stability using Lyapunov function techniques shows the Lyapunov function evaluated at the solution process goes to 0 as time $t \rightarrow \infty$. A distinctive feature of this paper is to obtain estimates of path-wise rates of convergence, which pinpoints how fast the aforementioned convergence to 0 taking place. Finally, some examples are given to illustrate our findings.
\end{abstract}

Keywords. Switching diffusion, past-dependent switching, existence and uniqueness of solution, Feller property.

Subject Classification. 34D20, 60H10, 93D05, 93D20.

\footnotetext{
${ }^{*}$ This research was supported in part by the National Science Foundation under grant DMS-1207667.

${ }^{\dagger}$ Department of Mathematics, Wayne State University, Detroit, MI 48202, dangnh.maths@gmail.com.

${ }^{\ddagger}$ Department of Mathematics, Wayne State University, Detroit, MI 48202, gyin@math.wayne.edu.
} 


\section{Introduction}

In the new era, because of the pressing needs in networked systems (including physical, biological, ecological, and social dynamic systems), large-scale optimization, and wired and wireless communications, many new sophisticated control systems have come into being. Hybrid systems in which discrete and continuous states coexist and interact, are such a representative. In particular, taking random disturbances into consideration, the so-called regime-switching diffusion systems have drawn resurgent and increasing attentions. A regime-switching diffusion is a two-component process $(X(t), \alpha(t))$, a continuous component and a discrete component taking values in a set consisting of isolated points. When the discrete component takes a value $i$ (i.e., $\alpha(t)=i$ ), the continuous component $X(t)$ evolves according to the diffusion process whose drift and diffusion coefficients depend on $i$. Asymptotic properties of such systems such as stability have been studied intensively, because of numerous applications. For example, many issues such as permanence, extinction, and persistence etc. of species in population dynamics and ecology are all linked to the stability issues.

Because many systems are in operation for a long period of time, an important problem of great interest is the stability of such systems. Many results on different types of stability have been given for switching diffusions when the state space of $\alpha(t)$ is finite (e.g., [7, 10, 17, 20, 21]). Assuming that $\alpha(t)$ takes values in a countable state space, stability of the processes is more difficult to analyze. To the best of our knowledge, very few papers have considered stability of switching diffusion with countable switching states. In [13], some conditions for stability of those systems have been given by approximating the generator of continuous state dependent switching process by that of a Markov chain with finite state space.

To find sufficient conditions for stability, it is desirable to find some common threads that are shared by many specific systems. Our motivation is based on the following thoughts. First, although the dynamics of $X(t)$ depend on the residence of the state of $\alpha(t)$, the structures of equations for different states of $\alpha(t)$ are not drastically different but rather similar in certain sense. This observation suggests finding a Lyapunov function that has similar form in different states of $\alpha(t)$. For instance, suppose there is a Lyapunov function $V(x)$ such that in each discrete state $i$, we have $\mathcal{L}_{i} V(x) \leq c_{i} V(x)$, where $\mathcal{L}_{i}$ is the generator of the diffusion in regime $i$ (more conditions and explanations for this inequality and related issues will be given in the next sections). In this case, there is a common Lyapunov function shared by all the discrete states (or the Lyapunov function is independent of the discrete states). It is well known that the sign of $c_{i}$ determines stability of the diffusion in each state $i$. For the regime-switching diffusion, one can expect that the stability of the system depends not only on $\left\{c_{i}\right\}$ but also on the generator $Q(x)$ of the switching part. A natural question is: under what relation between $\left\{c_{i}\right\}$ and $Q(x)$, the regime-switching diffusion is stable? When the number of regimes is finite, this question has been answered relatively completely (see [7, 13]). However, it is not straightforward to answer this question for the case of the discrete states belonging to a countable state space. We aim to take the challenges here. Moreover, this paper also considers a generalization when the condition $\mathcal{L}_{i} V(x) \leq c_{i} V(x)$ is replaced with a condition of the type $\mathcal{L}_{i} V(x) \leq c_{i} g(V(x))$.

To date, muck work has been devoted to the asymptotic stability of diffusions and switching diffusions. A commonly used technique is based on Lyapunov stability argument. For example, treating asymptotic stability, much effort has been devoted to obtaining sufficient conditions under which the Lyapunov function evaluated at the solutions of the processes go to 0 as $t \rightarrow \infty$. However, the question on how fast the Lyapunov function goes to 0 is unknown to date to the best of our knowledge. The current paper settles this issue; it may be one of the first to provide a convergence rate of the underlying process. As another novel contribution, we estimate the convergence rate of the solution to the equilibrium point by use of properties of the function $g(\cdot)$. 
Treating switching diffusions as Markov processes, one may obtain sufficient conditions for stability by using a Lyapunov function satisfying certain properties. However, the conditions are often not directly related to the given system coefficients (such as the drifts and diffusion matrices). To obtain conditions that are based on coefficients of the systems, we look at the issue of linearized (about the point of equilibrium) of the systems. The idea is originated from the topological equivalence of the linearized systems and original nonlinear systems due to the well-known Hartman-Grobman theorem in differential equations. Here in addition to linearizing the systems about the equilibrium point, we also replace $Q(x)$ by $Q(0)$.

The rest of this paper is organized as follows. In Section 2, we formulate the equation for a regime-switching diffusion and pose appropriate conditions for the existence and uniqueness of solutions. We then provide the definitions of certain types of stability as well as give general conditions for the stability of switching. In Section 3 , novel and practical conditions for stability and instability of regime-switching diffusions are given. Applications of these conditions to linearizable systems are given in Section 4 and examples are provided in Section 5 to illustrate our findings. Section 6 is devoted to several remarks. Finally, we provide the proofs of a number of technical results in an appendix.

\section{Formulation and Auxiliary Results}

Let $\left(\Omega, \mathcal{F},\left\{\mathcal{F}_{t}\right\}_{t \geq 0}, \mathbb{P}\right)$ be a complete filtered probability space with the filtration $\left\{\mathcal{F}_{t}\right\}_{t \geq 0}$ satisfying the usual condition, i.e., it is increasing and right continuous while $\mathcal{F}_{0}$ contains all $\mathbb{P}$-null sets. Let $W(t)$ be an $\mathcal{F}_{t^{-}}$adapted and $\mathbb{R}^{d}$-valued Brownian motion. Suppose $b(\cdot, \cdot): \mathbb{R}^{n} \times \mathbb{Z}_{+} \rightarrow \mathbb{R}^{n}$ and $\sigma(\cdot, \cdot): \mathbb{R}^{n} \times \mathbb{Z}_{+} \rightarrow \mathbb{R}^{n \times d}$. Consider the two-component process $(X(t), \alpha(t))$, where $\alpha(t)$ is a pure jump process taking value in $\mathbb{Z}_{+}=\mathbb{N} \backslash\{0\}=\{1,2, \ldots\}$, the set of positive integers, and $X(t) \in \mathbb{R}^{n}$ satisfies

$$
d X(t)=b(X(t), \alpha(t)) d t+\sigma(X(t), \alpha(t)) d W(t)
$$

We assume that the jump intensity of $\alpha(t)$ depends on the current state of $X(t)$, that is, there are functions $q_{i j}(\cdot): \mathbb{R}^{n} \rightarrow \mathbb{R}$ for $i, j \in \mathbb{Z}_{+}$satisfying

$$
\begin{aligned}
& \mathbb{P}\{\alpha(t+\Delta)=j \mid \alpha(t)=i, X(s), \alpha(s), s \leq t\}=q_{i j}(X(t)) \Delta+o(\Delta) \text { if } i \neq j \text { and } \\
& \mathbb{P}\{\alpha(t+\Delta)=i \mid \alpha(t)=i, X(s), \alpha(s), s \leq t\}=1-q_{i}(X(t)) \Delta+o(\Delta) .
\end{aligned}
$$

Throughout this paper, $q_{i j}(x) \geq 0$ for each $i \neq j$ and $\sum_{j \in \mathbb{Z}_{+}} q_{i j}(x)=0$ for each $i$ and all $x \in \mathbb{R}^{n}$. Denote $q_{i}(x)=\sum_{j=1, j \neq i}^{\infty} q_{i j}(x)$ (so $\left.q_{i i}(x)=-q_{i}(x)\right)$. and $Q(x)=\left(q_{i j}(x)\right)_{\mathbb{Z}_{+} \times \mathbb{Z}_{+}}$. The process $\alpha(t)$ can be defined rigorously as the solution to a stochastic differential equation with respect to a Poisson random measure. For each function $x \in \mathbb{R}^{n}, i \in \mathbb{Z}_{+}$, let $\Delta_{i j}(x), j \neq i$ be the consecutive left-closed, right-open intervals of the real line, each having length $q_{i j}(x)$. That is,

$$
\begin{aligned}
& \Delta_{i 1}(x)=\left[0, q_{i 1}(x)\right), \\
& \Delta_{i j}(x)=\left[\sum_{k=1, k \neq i}^{j-1} q_{i k}(x), \sum_{k=1, k \neq i}^{j} q_{i k}(x)\right), j>1, j \neq i .
\end{aligned}
$$

Define $h: \mathbb{R}^{n} \times \mathbb{Z}_{+} \times \mathbb{R} \mapsto \mathbb{R}$ by $h(x, i, z)=\sum_{j=1, j \neq i}^{\infty}(j-i) \mathbf{1}_{\left\{z \in \Delta_{i j}(x)\right\}}$. The process $\alpha(t)$ can be defined as the solution to

$$
d \alpha(t)=\int_{\mathbb{R}} h\left(X_{t}, \alpha(t-), z\right) \mathfrak{p}(d t, d z)
$$


where $a(t-)=\lim _{s \rightarrow t^{-}} \alpha(s)$ and $\mathfrak{p}(d t, d z)$ is a Poisson random measure with intensity $d t \times \mathfrak{m}(d z)$ and $\mathfrak{m}$ is the Lebesgue measure on $\mathbb{R}$ such that $\mathfrak{p}(d t, d z)$ is independent of the Brownian motion $W(\cdot)$. The pair $(X(t), \alpha(t))$ is therefore a solution to

$$
\left\{\begin{array}{l}
d X(t)=b(X(t), \alpha(t)) d t+\sigma(X(t), \alpha(t)) d W(t) \\
d \alpha(t)=\int_{\mathbb{R}} h(X(t), \alpha(t-), z) \mathfrak{p}(d t, d z) .
\end{array}\right.
$$

A strong solution to (2.3) on $[0, T]$ with initial data $(x, i) \in \mathbb{R}^{n} \times \mathbb{Z}_{+}$is an $\mathcal{F}_{t^{-}}$adapted process $(X(t), \alpha(t))$ such that

- $X(t)$ is continuous and $\alpha(t)$ is cadlag (right continuous with left limits) with probability 1 (w.p.1).

- $X(0)=x$ and $\alpha(0)=i_{0}$

- $(X(t), \alpha(t))$ satisfies (2.3) for all $t \in[0, T]$ w.p.1.

Let $f(\cdot, \cdot): \mathbb{R}^{n} \times \mathbb{Z}_{+} \mapsto \mathbb{R}$ be twice continuously differentiable in $x$. We define the operator $\mathcal{L} f(\cdot, \cdot): \mathbb{R}^{n} \times \mathbb{Z}_{+} \mapsto \mathbb{R}$ by

$$
\begin{aligned}
\mathcal{L} f(x, i) & =[\nabla f(x, i)]^{\top} b(x, i)+\frac{1}{2} \operatorname{tr}\left(\nabla^{2} f(x, i) A(x, i)\right)+\sum_{j=1, j \neq i}^{\infty} q_{i j}(x)[f(x, j)-f(x, i)] \\
& =\sum_{k=1}^{n} b_{k}(x, i) f_{k}(x, i)+\frac{1}{2} \sum_{k, l=1}^{n} a_{k l}(x, i) f_{k l}(x, i)+\sum_{j=1, j \neq i}^{\infty} q_{i j}(x)[f(x, j)-f(x, i)],
\end{aligned}
$$

where $\nabla f(x, i)=\left(f_{1}(x, i), \ldots, f_{n}(x, i)\right) \in \mathbb{R}^{1 \times n}$ and $\nabla^{2} f(x, i)=\left(f_{i j}(x, i)\right)_{n \times n}$ are the gradient and Hessian of $f(x, i)$ with respect to $x$, respectively, with

$$
\begin{aligned}
& f_{k}(x, i)=\left(\partial / \partial x_{k}\right) f(x, i), f_{k l}(x, i)=\left(\partial^{2} / \partial x_{k} \partial x_{l}\right) f(x, i), \text { and } \\
& A(x, i)=\left(a_{i j}(x, i)\right)_{n \times n}=\sigma(x, i) \sigma^{\top}(x, i),
\end{aligned}
$$

where $z^{\top}$ denotes the transpose of $z$. If $(X(t), \alpha(t))$ satisfies (2.3), then by modifying the proof of [14. Lemma 3, p.104], we have the generalized Itô formula:

$$
f(X(t), \alpha(t))-f(X(0), \alpha(0))=\int_{0}^{t} \mathcal{L} f(X(s), \alpha(s-)) d s+M_{1}(t)+M_{2}(t)
$$

where $M_{1}(\cdot)$ and $M_{2}(\cdot)$ are two local martingales defined by

$$
\begin{aligned}
& M_{1}(t)=\int_{0}^{t} \nabla f(X(s), \alpha(s-)) \sigma(X(s), \alpha(s-)) d W(s), \\
& M_{2}(t)=\int_{0}^{t} \int_{\mathbb{R}}[f(X(s), \alpha(s-)+h(X(s), \alpha(s-), z))-f(X(s), \alpha(s-))] \mu(d s, d z),
\end{aligned}
$$

and $\mu(d s, d z)$ is the compensated Poisson random measure given by

$$
\mu(d s, d z)=\mathfrak{p}(d s, d z)-m(d z) d s .
$$

Throughout this paper, we assume that either one of the following assumptions are satisfied. Under either of them, it is proved in [11] that (2.3) has a unique solution with given initial data. Moreover, the solution is a Markov-Feller process. 


\section{Assumption 2.1.}

1. For each $i \in \mathbb{Z}_{+}, H>0$, there is a positive constant $L_{i, H}$ such that

$$
|b(x, i)-b(y, i)|+|\sigma(y, i)-\sigma(x, i)| \leq L_{i, H}|x-y|
$$

if $x, y \in \mathbb{R}^{n}$ and $|x|,|y| \leq H$.

2. For each $i \in \mathbb{Z}_{+}$, there is a positive constant $\widetilde{L}_{i}$ such that

$$
|b(x, i)|+|\sigma(x, i)| \leq \widetilde{L}_{i}(|x|+1) .
$$

3. $q_{i j}(x)$ is continuous in $x \in \mathbb{R}^{n}$ for each $(i, j) \in \mathbb{Z}_{+}^{2}$. Moreover,

$$
M:=\sup _{x \in \mathbb{R}^{n}, i \in \mathbb{Z}_{+}}\left\{\left|q_{i}(x)\right|\right\}<\infty .
$$

\section{Assumption 2.2 .}

1. For each $i \in \mathbb{Z}_{+}, H>0$, there is a positive constant $L_{i, H}$ such that

$$
|b(x, i)-b(y, i)|+|\sigma(x, i)-\sigma(y, i)| \leq L_{i, H}|x-y|
$$

if $x, y \in \mathbb{R}^{n}$ and $|x|,|y| \leq H$.

2. There is a positive constant $\widetilde{L}$ such that

$$
|b(x, i)|+|\sigma(x, i)| \leq \widetilde{L}(|x|+1) .
$$

3. $q_{i j}(x)$ is continuous in $x \in \mathbb{R}^{n}$ for each $(i, j) \in \mathbb{Z}_{+}^{2}$. Moreover, for any $H>0$,

$$
M_{H}:=\sup _{x \in \mathbb{R}^{n},|x| \leq H, i \in \mathbb{Z}_{+}}\left\{\left|q_{i}(x)\right|\right\}<\infty .
$$

We suppose that $b(0, i)=0, \sigma(0, i)=0, i \in \mathbb{Z}_{+}$and give the following definitions of stability.

Definition 2.1. The trivial solution $X(t) \equiv 0$ is said to be

- stable in probability, if for any $h>0$,

$$
\lim _{x \rightarrow 0} \inf _{i \in \mathbb{Z}_{+}} \mathbb{P}_{x, i}\{X(t) \leq h \forall t \geq 0\}=1 .
$$

- asymptotic stable in probability, if it is stable in probability and

$$
\lim _{x \rightarrow 0} \inf _{i \in \mathbb{Z}_{+}} \mathbb{P}_{x, i}\left\{\lim _{t \rightarrow \infty} X(t)=0\right\}=1 .
$$

We state a general result that can be proved by well-known arguments; see [21, Section 7.2].

Theorem 2.2. Let $D$ be a neighborhood of $0 \in \mathbb{R}^{n}$. Suppose there exist three functions $V(x, i)$ : $D \times \mathbb{Z} \mapsto \mathbb{R}_{+}, \mu_{1}(x): D \mapsto \mathbb{R}_{+}, \mu_{2}(x): D \mapsto \mathbb{R}_{+}$such that

- $\mu_{1}(x), \mu_{2}(x)$ are continuous on $D, \mu_{k}(x)=0$ if and only if $x=0$ for $k=1,2$; 
- $V(x, i)$ is continuous on $D$ and twice continuously differentiable in $\mathcal{D} \backslash\{0\}$ for each $i \in \mathbb{Z}_{+}$;

- $\mu_{1}(x) \leq V(x, i)$ for any $(x, i) \in D \times \mathbb{Z}_{+}$.

Then the following conclusions hold.

- if $\mathcal{L} V(x, i) \leq 0$ for any $(x, i) \in D \times \mathbb{Z}_{+}$, the trivial solution is stable.

- if $\mathcal{L} V(x, i) \leq-\mu_{2}(x)$ for any $(x, i) \in D \times \mathbb{Z}_{+}$the trivial solution is asymptotically stable.

Let $\widehat{\alpha}(t)$ be the Markov chain with bounded generator $Q(0)$ and transition probability $\widehat{p}_{i j}(t)$

Definition 2.3. The Markov chain $\widehat{\alpha}(t)$ is said to be

- ergodic, if it has an invariant probability measure $\boldsymbol{\nu}=\left(\nu_{1}, \nu_{2}, \ldots\right)$ and

$$
\lim _{t \rightarrow \infty} \widehat{p}_{i j}(t)=\nu_{j} \text { for any } i, j \in \mathbb{Z}_{+}
$$

or equivalently,

$$
\lim _{t \rightarrow \infty} \sum_{j \in \mathbb{Z}_{+}}\left|\widehat{p}_{i j}(t)-\nu_{j}\right|=0 \text { for any } i \in \mathbb{Z}_{+}
$$

- strongly ergodic, if

$$
\lim _{t \rightarrow \infty} \sup _{i \in \mathbb{Z}_{+}}\left\{\sum_{j \in \mathbb{Z}_{+}}\left|\widehat{p}_{i j}(t)-\nu_{j}\right|\right\}=0 .
$$

- strongly exponentially ergodic, if there exist $C>0$ and $\lambda>0$ such that

$$
\sum_{j \in \mathbb{Z}_{+}}\left|\widehat{p}_{i j}(t)-\pi_{j}\right| \leq C e^{-\lambda t} \text { for any } i \in \mathbb{Z}_{+}, t \geq 0
$$

We refer to [1] for some properties and sufficient conditions for the aforementioned ergodicity.

\section{Certain Practical Conditions for Stability and Instability}

For each $h>0$, denote by $B_{h} \subset \mathbb{R}^{n}$ the open ball centered at 0 with radius $h$. Throughout this section, let $D$ be a neighborhood of 0 satisfying $D \subset B_{1}$. We also denote by $\widehat{\alpha}(t)$ the continuoustime Markov chain with generator $Q(0)$. Denote by $\mathcal{L}_{i}$ the generator of the diffusion when the discrete component is in state $i$, that is,

$$
\mathcal{L}_{i} V(x)=\nabla V(x) b(x, i)+\frac{1}{2} \operatorname{tr}\left(\nabla^{2} V(x) A(x, i)\right) .
$$

We first state a theorem, which generalizes [7, Theorem 4.3], a result for switching diffusions when the switching takes values in a finite set.

Theorem 3.1. Suppose that the Markov chain $\widehat{\alpha}(t)$ is strongly ergodic with invariant probability measure $\boldsymbol{\nu}=\left(\nu_{1}, \nu_{2}, \ldots\right)$ and that

$$
\sup _{i \in \mathbb{Z}_{+}} \sum_{j \neq i}\left|q_{i j}(x)-q_{i j}(0)\right| \rightarrow 0 \text { as } x \rightarrow 0 .
$$


Let $D$ be a neighborhood of 0 and $V: D \mapsto \mathbb{R}_{+}$satisfying that $V(x)=0$ if and only if $x=0$ and that $V(x)$ is continuous on $D$, twice continuously differentiable in $D \backslash\{0\}$. Suppose that there is a bounded sequence of real numbers $\left\{c_{i}: i \in \mathbb{Z}_{+}\right\}$such that

$$
\mathcal{L}_{i} V(x) \leq c_{i} V(x) \forall x \in D \backslash\{0\} .
$$

Then, if $\sum_{i \in \mathbb{Z}_{+}} c_{i} \nu_{i}<0$, the trivial solution is asymptotic stable in probability.

Proof. Let $\lambda=-\sum_{i \in \mathbb{Z}_{+}} c_{i} \nu_{i}$. Since $\sum_{i \in \mathbb{Z}_{+}} \nu_{i}=1$, we have $\sum_{i \in \mathbb{Z}_{+}}\left(c_{i}+\lambda\right) \nu_{i}=0$. Since $\widehat{\alpha}(t)$ is strongly ergodic, it follows from Lemma A.1 that there exists a bounded sequence of real numbers $\left\{\gamma_{i}: i \in \mathbb{Z}_{+}\right\}$such that

$$
\sum_{j \in \mathbb{Z}_{+}} q_{i j}(0) \gamma_{j}=\lambda+c_{i} \text { for any } i \in \mathbb{Z}_{+}
$$

Since $\sum_{j \in \mathbb{Z}_{+}} q_{i j}(0)=0$ for any $i \in \mathbb{Z}_{+}$it follows from (3.3) that

$$
\sum_{j \in \mathbb{Z}_{+}} q_{i j}(0) \tilde{\gamma}_{j}=\sum_{j \in \mathbb{Z}_{+}} q_{i j}(0)\left(1-p \gamma_{j}\right)=-p\left(\lambda+c_{i}\right) \text { for any } i \in \mathbb{Z}_{+}
$$

Since $\left\{\gamma_{i}\right\}$ is bounded, we can choose $p \in(0,1)$ such that

$$
p\left|\gamma_{i}\right| \leq \min \{0.25 \lambda, 0.5\}
$$

In view of (3.1) and (3.5), there is an $h>0$ sufficiently small such that

$$
\sum_{j \in \mathbb{Z}_{+}}\left(1-p \gamma_{j}\right)\left|q_{i j}(x)-q_{i j}(0)\right|<\frac{p \lambda}{4} \quad \forall x \in B_{h} .
$$

Define the function $U(x, i): B_{h} \times \mathbb{Z}_{+} \mapsto \mathbb{R}_{+}$by $U(x, i)=\left(1-p \gamma_{i}\right) V^{p}(x)$. By Itô's formula, (3.1), (3.4), and (3.6), we have

$$
\begin{aligned}
\mathcal{L} U(x, i) & =p\left(1-p \gamma_{i}\right) V^{p-1} \mathcal{L}_{i} V(x)-\frac{p(1-p)}{2} V^{p-2}\left|V_{x}(x) \sigma(x, i)\right|^{2}+V^{p}(x) \sum_{j \in \mathbb{Z}_{+}}\left(1-p \gamma_{j}\right) q_{i j}(x) \\
& \leq c_{i} p\left(1-p \gamma_{i}\right) V^{p-1}+V^{p}(x) \sum_{j \in \mathbb{Z}_{+}}\left(1-p \gamma_{j}\right) q_{i j}(0)+V^{p}(x) \sum_{j \in \mathbb{Z}_{+}}\left(1-p \gamma_{j}\right)\left|q_{i j}(x)-q_{i j}(0)\right| \\
& \leq c_{i} p\left(1-p \gamma_{i}\right) V^{p-1}-p\left(\lambda+c_{i}\right) V^{p}(x)+V^{p}(x) \sum_{j \in \mathbb{Z}_{+}}\left(1-p \gamma_{j}\right)\left|q_{i j}(x)-q_{i j}(0)\right| \\
& \leq p\left(-\lambda-p \gamma_{i}\right) V^{p}(x)+V^{p}(x) \sum_{j \in \mathbb{Z}_{+}}\left(1-p \gamma_{j}\right)\left|q_{i j}(x)-q_{i j}(0)\right| \\
& \leq-0.75 p \lambda V^{p}(x)+0.25 p \lambda V^{p}(x)=-0.5 p \lambda V^{p}(x) \text { for }(x, i) \in B_{h} \times \mathbb{Z}_{+} .
\end{aligned}
$$

By Theorem 2.2, it follows from (3.7) that the trivial solution is asymptotically stable.

The hypothesis of this theorem seems to be restrictive. It requires the strongly exponential ergodicity of $Q(0)$ and the uniform convergence to 0 of the sum $\sum_{j \neq i}\left|q_{i j}(x)-q_{i j}(0)\right|$. To treat cases in which $Q(0)$ is strongly ergodic (not exponentially ergodic) or even only ergodic, as well as to relax the condition (3.1), we need a more complicated method. Our method, which is inspired by the idea in [4, utilizes the ergodicity of $Q(0)$ and the analysis of the Laplace transform. Similar techniques of using the Laplace transform can also be seen in the large deviations theory and related 
applications [3, 22]. We also take a step further by estimating the pathwise rate of convergence of solutions.

Let $\Gamma$ be a family of increasing and continuously differentiable functions $g: \mathbb{R}_{+} \mapsto \mathbb{R}_{+}$such that $g(y)=0$ iff $y=0$. Since $\frac{d g}{d y}(y)$ is bounded on $[0,1]$ and $g(0)=0$, it is easy to show that the function

$$
G(y):=-\int_{y}^{h} \frac{d z}{g(z)} \quad \text { on }[0, h]
$$

is non-positive and strictly decreasing and $\lim _{y \rightarrow 0} G(y)=-\infty$. Its inverse $G^{-1}:(-\infty, 0] \mapsto(0, h]$ satisfies

$$
\lim _{t \rightarrow \infty} G^{-1}(-t)=0 .
$$

We state some assumptions to be used in what follows; we will also provide some lemmas whose proofs are relegated to the appendix.

Assumption 3.1. There are functions $g \in \Gamma, V: D \mapsto \mathbb{R}_{+}$such that

- $V(x)=0$ if and only if $x=0$

- $V(x)$ is continuous on $D$ and twice continuously differentiable in $D \backslash\{0\}$.

- there is a bounded sequence of real numbers $\left\{c_{i}: i \in \mathbb{Z}_{+}\right\}$such that

$$
\mathcal{L}_{i} V(x) \leq c_{i} g(V(x)) \forall x \in D \backslash\{0\} .
$$

Lemma 3.1. Under Assumption [3.1, For any $\varepsilon, T, h>0$, there exists an $\tilde{h}=\tilde{h}(\varepsilon, T, h)$ such that

$$
\mathbb{P}_{x, i}\left\{\tau_{h} \geq T\right\}<\varepsilon, \quad \text { for all }(x, i) \in B_{\tilde{h}} \times \mathbb{Z}_{+}
$$

where $\tau_{h}=\inf \{t \geq 0:|X(t)| \geq h\}$.

Lemma 3.2. Let $Y$ be a random variable, $\theta_{0}>0$ a constant, and suppose

$$
\mathbb{E} \exp \left(\theta_{0} Y\right)+\mathbb{E} \exp \left(-\theta_{0} Y\right) \leq K_{1}
$$

Then the log-Laplace transform $\phi(\theta)=\ln \mathbb{E} \exp (\theta Y)$ is twice differentiable on $\left[0, \frac{\theta_{0}}{2}\right)$ and

$$
\frac{d \phi}{d \theta}(0)=\mathbb{E} Y, \quad \text { and } 0 \leq \frac{d^{2} \phi}{d \theta^{2}}(\theta) \leq K_{2}, \theta \in\left[0, \frac{\theta_{0}}{2}\right)
$$

for some $K_{2}>0$. As a result of Taylor's expansion, we have

$$
\phi(\theta) \leq \theta \mathbb{E} Y+\theta^{2} K_{2}, \quad \text { for } \theta \in\left[0,0.5 \theta_{0}\right) .
$$

Lemma 3.3. Under the assumption $b(0, i)=0, \sigma(0, i)=0, i \in \mathbb{Z}_{+}$, we have

$$
\mathbb{P}_{x, i}\{X(t)=0 \text { for some } t \geq 0\}=0 \text { for any } x \neq 0, i \in \mathbb{Z}_{+} \text {. }
$$

With the auxiliary results above, we can prove our main results. 
Theorem 3.2. Suppose that the Markov chain $\widehat{\alpha}(t)$ is ergodic with invariant probability measure $\boldsymbol{\nu}=\left(\nu_{1}, \nu_{2}, \ldots\right)$ and Assumption 3.1 is satisfied with additional conditions:

$$
\limsup _{i \rightarrow \infty} c_{i}<0
$$

and

$$
M_{g}:=\sup _{0<|x|<h, i \in \mathbb{Z}_{+}}\left\{\left|\frac{V_{x}(x) \sigma(x, i)}{g(V(x))}\right|\right\}<\infty .
$$

Then, if $\sum_{i \in \mathbb{Z}_{+}} c_{i} \nu_{i}<0$, the trivial solution is asymptotic stable in probability, that is, for any $h>0$ such that $B_{h} \subset D$, and $\varepsilon>0$, there exists $\delta=\delta(h, \varepsilon)>0$ such that

$$
\mathbb{P}_{x, i}\left\{X(t)<h \forall t \geq 0, \quad \text { and } \quad \lim _{t \rightarrow \infty} X(t)=0\right\}>1-\varepsilon \text { for any }(x, i) \in B_{\delta} \times \mathbb{Z}_{+} .
$$

Moreover, there is a $\lambda>0$ such that

$$
\mathbb{P}_{x, i}\left\{\lim _{t \rightarrow \infty} \frac{V(X(t))}{G^{-1}(-\lambda t)} \leq 1\right\}>1-\varepsilon \text { for any }(x, i) \in B_{\delta} \times \mathbb{Z}_{+} .
$$

Remark 3.1. Before proceeding to the proof of the theorem, let us make a brief comment. In addition to providing sufficient conditions for asymptotic stability, a significant new element here is the rate of convergence given in (3.12). Although there are numerous treatment of stochastic stability by a host of authors for diffusions and switching diffusions. The rate result in Theorem 3.2 appears to the first one of its kind.

Proof. The proof is divided into two steps. We first show the trivial solution is stable in probability and then we prove asymptotic stability and estimate the path-wise convergence rate.

\section{Step 1: Stability.}

Let $h>0$ such that $B_{h} \subset D$. Since $\left\{c_{i}\right\}$ is bounded,

$$
\lim _{k \rightarrow \infty} \sum_{i \leq k} c_{i} \nu_{i}=\sum_{i \in \mathbb{Z}_{+}} c_{i} \nu_{i}<0 .
$$

This and (3.10) show that there exists $k_{0} \in \mathbb{Z}_{+}$such that

$$
-\lambda_{1}:=\sum_{i \leq k_{0}} c_{i} \nu_{i}<0
$$

and

$$
-2 \lambda_{2}:=\sup _{i>k_{0}} c_{i}<0
$$

Let $\bar{c}=\sup _{i \in \mathbb{Z}_{+}}\left|c_{i}\right|$ and $m_{0}$ be an positive integer satisfying $m_{0} \lambda_{2}>\bar{c}+M_{g}+1$. Define $G(y)=$ $-\int_{y}^{h} g^{-1}(z) d z$. In view of Lemma 3.3, if $X(0) \neq 0$, then $X(t) \neq 0$ a.s, which leads to $g(V(X(t)) \neq 0$ a.s. Thus, we have from Itô's formula and the increasing property of $g(\cdot)$ that

$$
\begin{aligned}
G\left(V\left(X\left(\tau_{h} \wedge t\right)\right)\right)= & G(V(x))+\int_{0}^{\tau_{h} \wedge t} \frac{\mathcal{L}_{\alpha(s)} V(X(s))}{g(V(X(s)))} d s \\
& -\int_{0}^{\tau_{h} \wedge t} \frac{\frac{d g}{d y}(V(X(s)))\left|V_{x}(X(s)) \sigma(X(s), \alpha(s))\right|^{2}}{2 g^{2}(V(X(s)))} d s \\
& +\int_{0}^{\tau_{h} \wedge t} \frac{V_{x}(X(s)) \sigma(X(s), \alpha(s))}{g(V(X(s)))} d W(s) \leq G(V(x))+H(t),
\end{aligned}
$$


where

$$
H(t)=\int_{0}^{\tau_{h} \wedge t} c(\alpha(s)) d s+\int_{0}^{\tau_{h} \wedge t} \frac{V_{x}(X(s)) \sigma(X(s), \alpha(s))}{g(V(X(s)))} d W(s) .
$$

By Itô's formula,

$$
\begin{aligned}
e^{\theta H(t)}= & +\int_{0}^{t \wedge \tau_{h}} e^{\theta H(s)}\left[\theta c(\alpha(s))+\frac{\theta^{2}}{2} \frac{\left|V_{x}(X(s)) \sigma(X(s), \alpha(s))\right|^{2}}{2 g^{2}(V(X(s)))}\right] d s \\
& +\theta \int_{0}^{t \wedge \tau_{h}} e^{\theta H(s)} \frac{V_{x}(X(s)) \sigma(X(s), \alpha(s))}{g(V(X(s)))} d W(s),
\end{aligned}
$$

which leads to

$$
\begin{aligned}
\mathbb{E}_{x, i} e^{\theta H(t)} & =1+\mathbb{E}_{x, i} \int_{0}^{\tau_{h} \wedge t} e^{\theta H(s)}\left[\theta c(\alpha(s))+\frac{\theta^{2}}{2} \frac{\left|V_{x}(X(s)) \sigma(X(s), \alpha(s))\right|^{2}}{2 g^{2}(V(X(s)))}\right] d s \\
& \leq 1+\left[\bar{c}+M_{g}\right] \mathbb{E}_{x, i} \int_{0}^{\tau_{h} \wedge t} e^{\theta H(s)} d s \\
& \leq 1+\left[\bar{c}+M_{g}\right] \int_{0}^{t} \mathbb{E}_{x, i} e^{\theta H(s)} d s .
\end{aligned}
$$

In view of Gronwall's inequality, for any $t \geq 0$ and $(x, i) \in B_{h} \times \mathbb{Z}_{+}$, we have

$$
\mathbb{E}_{x, i} e^{\theta H(t)} \leq e^{\theta\left[\bar{c}+M_{g}\right] t}, \theta \in[-1,1] .
$$

On the other hand, we have

$$
\begin{aligned}
\mathbb{E}_{x, i} H(t) & \leq \mathbb{E}_{x, i} \int_{0}^{\tau_{h} \wedge t} c(\alpha(s)) d s \\
& \leq \mathbb{E}_{x, i} \int_{0}^{t} c(\alpha(s)) d s-\mathbb{E}_{x, i} \int_{\tau_{h} \wedge t}^{t} c(\alpha(s)) d s \\
& \leq \mathbb{E}_{x, i} \int_{0}^{t} c(\alpha(s)) d s+t \bar{c} \mathbb{P}_{x, i}\left\{\tau_{h}<t\right\} .
\end{aligned}
$$

Because of the ergodicity of $\widehat{\alpha}(t)$, there exists a $T>0$ depending on $k_{0}$ such that

$$
\mathbb{E}_{0, i} \int_{0}^{t} c(\alpha(s)) d s=\mathbb{E}_{i} \int_{0}^{t} c(\widehat{\alpha}(s)) d s \leq-\frac{3 \lambda_{1}}{4} t \quad \forall t \geq T, i \leq k_{0} .
$$

By the Feller property of $(X(t), \alpha(t))$ there exists an $h_{1} \in(0, h)$ such that

$$
\mathbb{E}_{x, i} \int_{0}^{t} c(\alpha(s)) d s \leq-\frac{\lambda_{1}}{2} t \quad \forall t \in\left[T, T_{2}\right],|x| \leq h_{1}, i \leq k_{0}
$$

where $T_{2}=\left(m_{0}+1\right) T$. In view of Lemma 3.1 , there exists an $h_{2} \in\left(0, h_{1}\right)$ such that

$$
\bar{c} \mathbb{P}_{x, i}\left\{\tau_{h}<m_{0} T\right\} \leq \frac{\lambda_{1}}{4} \text { provided }|x| \leq h_{2}, i \in \mathbb{Z}_{+} .
$$

Applying (3.19) and (3.20) to (3.17), we obtain

$$
\mathbb{E}_{x, i} H(t) \leq-\frac{\lambda_{1}}{4} t \text { if } 0<|x| \leq h_{2}, i \leq k_{0}, t \in\left[T, T_{2}\right]
$$


By Lemma 3.2, it follows from (3.16) and (3.21) that for $\theta \in[0,0.5], 0<|x|<h_{2}, i \leq k_{0}, t \in\left[T, T_{2}\right]$, we have

$$
\begin{aligned}
\ln \mathbb{E}_{x, i} e^{\theta H(t)} & \leq \theta \mathbb{E}_{x, i} H(t)+\theta^{2} K \\
& \leq-\theta \frac{\lambda_{1} t}{4}+\theta^{2} K
\end{aligned}
$$

for some $K>0$ depending on $T_{2}, \bar{c}$ and $M_{g}$. Let $\theta \in(0,0.5]$ such that

$$
\theta K<\frac{\lambda_{1} T}{8}, \text { and } \theta M_{g}<\lambda_{2}
$$

we have

$$
\ln \mathbb{E}_{x, i} e^{\theta H(t)} \leq-\frac{\theta \lambda_{1} t}{8} \text { for } 0<|x|<h_{2}, i \leq k_{0}, t \in\left[T, T_{2}\right]
$$

or equivalently,

$$
\mathbb{E}_{x, i} e^{\theta H(t)} \leq \exp \left\{-\frac{\theta \lambda_{1} t}{8}\right\} \text { for } 0<|x|<h_{2}, i \leq k_{0}, t \in\left[T, T_{2}\right]
$$

In what follows, we fix a $\theta>0$ satisfying (3.24). Exponentiating both sides of the inequality $G\left(V\left(X\left(\tau_{h} \wedge t\right)\right)\right) \leq G(V(x))+H(t)$ we have for $0<|x|<h_{2}, i \leq k_{0}, t \in\left[T, T_{2}\right]$ that

$$
\mathbb{E}_{x, i} U\left(X\left(\tau_{h} \wedge t\right)\right) \leq U(x) \mathbb{E}_{x, i} e^{\theta H(t)} \leq U(x) \exp \left\{-\frac{\theta \lambda_{1} t}{8}\right\}
$$

where $U(x)=\exp (\theta G(V(x)))$. Since $\lim _{x \rightarrow 0} G(V(x))=-\infty$ then

$$
\lim _{x \rightarrow 0} U(x)=0 .
$$

Using the inequality $G\left(V\left(X\left(\tau_{h} \wedge t\right)\right)\right) \leq G(V(x))+H(t)$ and (3.16) we have

$$
\mathbb{E}_{x, i} U\left(X\left(\tau_{h} \wedge t\right)\right) \leq U(x) \exp \left\{\theta\left[\bar{c}+M_{g}\right] t\right\} \text {, for all }(x, i) \in B_{h} \times \mathbb{Z}_{+}, t \geq 0 .
$$

Now, let $\Delta=\inf \left\{U(x): h_{2} \leq|x| \leq h\right\}>0$. Define stopping times

$$
\xi=\inf \left\{t \geq 0: \alpha(t) \leq k_{0}\right\}, \quad \text { and } \zeta=\inf \{t \geq 0: U(X(t)) \geq \Delta\} .
$$

Clearly, if $X(0) \in B_{h}$ then $\zeta \leq \tau_{h}$ and if $t<\zeta$ then $|X(t)|<h_{2}$. By computation and (3.23), we have

$$
\begin{aligned}
\mathcal{L}_{i} U(x) \leq \theta U(x)\left[c_{i}+\left[\theta-\dot{g}(V(x)] \frac{\left|V_{x}(x) \sigma(x, i)\right|^{2}}{g(V(x))}\right]\right. & \leq \theta\left(-2 \lambda_{2}+\theta M_{g}\right) U(x) \\
& \leq-\theta \lambda_{2} U(x), \text { for } 0<|x|<h, i>k_{0} .
\end{aligned}
$$

It follows from Itô's formula that

$$
\begin{aligned}
\mathbb{E}_{x, i} e^{\theta \lambda_{2}(t \wedge \xi \wedge \zeta)} U(X(t \wedge \xi) & =U(x)+\mathbb{E}_{x, i} \int_{0}^{t \wedge \xi \wedge \zeta} e^{\lambda_{2} s}\left[\theta \lambda_{2} U(X(s))+\mathcal{L}_{\alpha(t)} U(X(s))\right] d s \\
& \leq U(x), \text { for } 0<|x|<h, i \in \mathbb{Z}_{+} .
\end{aligned}
$$


We have the following estimate for $0<|x|<h, i>k_{0}$.

$$
\begin{aligned}
\mathbb{E}_{x, i} e^{\theta \lambda_{2}\left(T_{2} \wedge \xi \wedge \zeta\right)} U\left(X\left(T_{2} \wedge \xi \wedge \zeta\right)\right)= & \mathbb{E}_{x, i} \mathbf{1}_{\left\{\xi \wedge \zeta<m_{0} T\right\}} e^{\theta \lambda_{2}\left(T_{2} \wedge \xi \wedge \zeta\right)} U\left(X\left(T_{2} \wedge \xi \wedge \zeta\right)\right) \\
& +\mathbb{E}_{x, i} \mathbf{1}_{\left\{m_{0} T \leq \xi \wedge \zeta<T_{2}\right\}} e^{\theta \lambda_{2}\left(T_{2} \wedge \xi \wedge \zeta\right)} U\left(X\left(T_{2} \wedge \xi \wedge \zeta\right)\right) \\
& +\mathbb{E}_{x, i} \mathbf{1}_{\left\{\xi \wedge \zeta \geq T_{2}\right\}} e^{\theta \lambda_{2}\left(T_{2} \wedge \xi \wedge \zeta\right)} U\left(X\left(T_{2} \wedge \xi \wedge \zeta\right)\right) \\
\geq & \mathbb{E}_{x, i} \mathbf{1}_{\left\{\xi \wedge \zeta \leq m_{0} T\right\}} U(X(\xi \wedge \zeta)) \\
& +e^{\theta \lambda_{2} m_{0} T} \mathbb{E}_{x, i} \mathbf{1}_{\left\{m_{0} T \leq \xi \wedge \zeta<T_{2}\right\}} U(X(\xi \wedge \zeta)) \\
& +e^{\theta \lambda_{2} T_{2}} \mathbb{E}_{x, i} \mathbf{1}_{\left\{\xi \geq T_{2}\right\}} U\left(X\left(T_{2}\right)\right) .
\end{aligned}
$$

Since $\mathbb{P}_{x, i}\{\zeta=0\}=1$ if $i \leq k_{0}$, (3.29) holds for $0<|x|<h, i \in \mathbb{Z}_{+}$. Noting that $U(x) \wedge \Delta \leq \Delta$ for any $x \in B_{h}$, we have

$$
\mathbb{E}\left[U\left(X\left(T_{2} \wedge \tau_{h}\right)\right) \wedge \Delta \mid \zeta<m_{0} T, \zeta \leq \xi\right] \leq \Delta \leq U(X(\zeta))=U(X(\xi \wedge \zeta)) .
$$

If $\xi<\zeta$, then $U(X(\xi))<\Delta$. By strong Markov property of $(X(t), \alpha(t))$, (3.25), and (3.16), we have

$$
\mathbb{E}\left[U\left(X\left(T_{2} \wedge \tau_{h}\right)\right) \wedge \Delta \mid \xi<m_{0} T \wedge \zeta\right] \leq U(X(\xi))=U(X(\xi \wedge \zeta))
$$

and

$$
\mathbb{E}\left[U\left(X\left(T_{2} \wedge \tau_{h}\right)\right) \wedge \Delta \mid m_{0} T \leq \xi<T_{2} \wedge \zeta\right] \leq U(X(\xi)) e^{\theta\left(\bar{c}+M_{g}\right) T}=U(X(\xi \wedge \zeta)) e^{\theta\left(\bar{c}+M_{g}\right) T} .
$$

From the three estimates above, we have

$$
\begin{aligned}
\mathbb{E}_{x, i} \mathbf{1}_{\left\{\xi \wedge \zeta \leq m_{0} T\right\}}\left[U\left(X\left(T_{2} \wedge \tau_{h}\right)\right) \wedge \Delta\right]= & \mathbb{E}_{x, i} \mathbf{1}_{\left\{\zeta<m_{0} T, \zeta \leq \xi\right\}}\left[U\left(X\left(T_{2} \wedge \tau_{h}\right)\right) \wedge \Delta\right] \\
& +\mathbb{E}_{x, i} \mathbf{1}_{\left\{\xi<m_{0} T \wedge \zeta\right\}}\left[U\left(X\left(T_{2} \wedge \tau_{h}\right)\right) \wedge \Delta\right] \\
\leq & \mathbb{E}_{x, i} \mathbf{1}_{\left\{\xi \wedge \zeta<m_{0} T\right\}} U(X(\xi \wedge \zeta)) .
\end{aligned}
$$

Similarly,

$$
\begin{aligned}
\mathbb{E}_{x, i} \mathbf{1}_{\left\{m_{0} T \leq \xi \wedge \zeta<T_{2}\right\}}\left[U\left(X\left(T_{2} \wedge \tau_{h}\right)\right) \wedge \Delta\right] & \leq e^{\theta\left(\bar{c}+M_{g}\right) T} \mathbb{E}_{x, i} \mathbf{1}_{\left\{m_{0} T \leq \xi \wedge \zeta<T_{2}\right\}} U(X(\xi \wedge \zeta)) \\
& \leq e^{\theta \lambda_{2} m_{0} T} \mathbb{E}_{x, i} \mathbf{1}_{\left\{m_{0} T \leq \xi \wedge \zeta<T_{2}\right\}} U(X(\xi \wedge \zeta)),
\end{aligned}
$$

where the last line follows from $m_{0} \lambda_{2}>\bar{c}+M_{g}+1$. Applying (3.30) and (3.31) to (3.29), we obtain

$$
\mathbb{E}_{x, i}\left[U\left(X\left(T_{2} \wedge \tau_{h}\right)\right) \wedge \Delta\right] \leq U(x) \text { for any }(x, i) \in B_{h} \times \mathbb{Z} .
$$

Since $\mathbb{E}_{x, i}\left[U\left(X\left(T_{2} \wedge \tau_{h}\right)\right) \wedge \Delta\right] \leq \Delta$, we have

$$
\mathbb{E}_{x, i}\left[U\left(X\left(T_{2} \wedge \tau_{h}\right)\right) \wedge \Delta\right] \leq U(x) \wedge \Delta \text { for any }(x, i) \in B_{h} \times \mathbb{Z} .
$$

This together with the Markov property of $(X(t), \alpha(t))$ implies that $\left\{M(k):=\left[U\left(X\left(k T_{2} \wedge \tau_{h}\right)\right) \wedge\right.\right.$ $\left.\Delta], k \in \mathbb{Z}_{+}\right\}$is a super-martingale. Let $\eta=\inf \left\{k \in \mathbb{Z}_{+}: M(k)=\Delta\right\}$. Clearly, $\{\eta<\infty\} \supset\left\{\tau_{h}<\right.$ $\infty\}$. For any $\varepsilon>0$, if $U(x)<\varepsilon \Delta$ we have that

$$
\mathbb{P}_{x, i}\{\eta<k\} \leq \frac{\mathbb{E}_{x, i} M(\eta \wedge k)}{\Delta} \leq \frac{U(x)}{\Delta} \leq \varepsilon
$$

Letting $k \rightarrow \infty$ we have

$$
\mathbb{P}_{x, i}\left\{\tau_{h}<\infty\right\} \leq \mathbb{P}_{x, i}\{\eta<\infty\} \leq \varepsilon \text {, if } U(x)<\varepsilon \Delta .
$$


We complete the proof of this step by noting that $\{x: U(x)<\varepsilon \Delta\}$ is a neighborhood of $x$ due to the fact that $\lim _{x \rightarrow 0} U(x)=0$.

Step 2: Asymptotic stability and pathwise convergence rate.

To prove the asymptotic stability in probability, we fix $h>0$ and define $U(x), T_{2}, m_{0}, \Delta$ depending on $h$ as in the first step. By virtue of (3.29), we have

$$
\begin{aligned}
\mathbb{E}_{x, i} e^{\theta \lambda_{2}\left(T_{2} \wedge \xi \wedge \zeta\right)} U\left(X\left(T_{2} \wedge \xi \wedge \zeta\right)\right) \geq & \mathbb{E}_{x, i} \mathbf{1}_{\left\{\xi \wedge \zeta<m_{0} T\right\}} U(X(\xi \wedge \zeta)) \\
& +e^{\theta \lambda_{2} m_{0} T} \mathbb{E}_{x, i} \mathbf{1}_{\left\{m_{0} T \leq \xi \wedge \zeta<T_{2}\right\}} U(X(\xi \wedge \zeta)) \\
& +e^{\theta \lambda_{2} T_{2}} \mathbb{E}_{x, i} \mathbf{1}_{\left\{\xi \wedge \zeta \geq T_{2}\right\}} U\left(X\left(T_{2}\right)\right) \\
\geq & \mathbb{E}_{x, i} \mathbf{1}_{\left\{\xi<m_{0} T, \zeta>\xi\right\}} U(X(\xi)) \\
& +e^{\theta \lambda_{2} m_{0} T} \mathbb{E}_{x, i} \mathbf{1}_{\left\{m_{0} T \leq \xi<T_{2}, \zeta>\xi\right\}} U(X(\xi)) \\
& +e^{\theta \lambda_{2} T_{2}} \mathbb{E}_{x, i} \mathbf{1}_{\left\{\xi \wedge \zeta \geq T_{2}\right\}} U\left(X\left(T_{2}\right)\right) .
\end{aligned}
$$

Recalling that $\zeta \leq \tau_{h}$ and $X(t)<h_{2}$ if $t<\zeta$, we have from (3.24) and (3.27) that

$$
\begin{aligned}
\mathbb{E}_{x, i} \mathbf{1}_{\left\{\zeta \geq T_{2}\right\}} \mathbf{1}_{\left\{\xi<m_{0} T\right\}} U\left(X\left(T_{2}\right)\right) & \leq \mathbb{E}_{x, i} \mathbf{1}_{\{\xi<\zeta\}} \mathbf{1}_{\left\{\xi<m_{0} T\right\}} U\left(X\left(T_{2} \wedge \tau_{h}\right)\right) \\
& \leq \mathbb{E}_{x, i}\left[\mathbf{1}_{\left\{\xi<m_{0} T \wedge \zeta\right\}} U(X(\xi)) \exp \left\{-\theta \frac{\lambda}{8}\left(T_{2}-\xi\right)\right\}\right] \\
& \leq \exp \left\{-\frac{\theta \lambda T}{8}\right\} \mathbb{E}_{x, i}\left[\mathbf{1}_{\{\zeta \geq \xi\}} \mathbf{1}_{\left\{\xi<m_{0} T\right\}} U(X(\xi))\right]
\end{aligned}
$$

and

$$
\begin{aligned}
\mathbb{E}_{x, i} \mathbf{1}_{\left\{m_{0} T \leq \xi<T_{2}, \zeta>T_{2}\right\}} U\left(X\left(T_{2}\right)\right) & \leq \mathbb{E}_{x, i} \mathbf{1}_{\left\{m_{0} T \leq \xi<T_{2} \wedge \zeta\right\}} U\left(X\left(T_{2} \wedge \tau_{h}\right)\right) \\
& \leq \mathbb{E}_{x, i}\left[\mathbf{1}_{\left\{m_{0} T \leq \xi<T_{2} \wedge \zeta\right\}} U(X(\xi)) \exp \left\{\theta\left(\bar{c}+M_{g}\right)\left(T_{2}-\xi\right)\right\}\right] \\
& \leq \exp \left\{\theta\left(\bar{c}+M_{g}\right) T\right\} \mathbb{E}_{x, i}\left[\mathbf{1}_{\left\{m_{0} T \leq \xi<T_{2} \wedge \zeta\right\}} U(X(\xi))\right] \\
& \leq \exp \{-\theta T\} \exp \left\{\theta \lambda_{2} m_{0} T\right\} \mathbb{E}_{x, i}\left[\mathbf{1}_{\left\{m_{0} T \leq \xi<T_{2} \wedge \zeta\right\}} U(X(\xi))\right] .
\end{aligned}
$$

On the other hand, we can write

$$
\mathbb{E}_{x, i} \mathbf{1}_{\left\{\xi \wedge \zeta \geq T_{2}\right\}} U\left(X\left(T_{2}\right)\right)=e^{-\theta \lambda_{2} T_{2}} e^{\theta \lambda_{2} T_{2}} \mathbb{E}_{x, i} \mathbf{1}_{\left\{\xi \wedge \zeta \geq T_{2}\right\}} U\left(X\left(T_{2}\right)\right) .
$$

Letting $p=\max \left\{\exp \left\{-\frac{\theta \lambda T}{8}\right\}, \exp \{-\theta T\}, \exp \left\{-\theta \lambda_{2} T_{2}\right\}\right\}<1$ and adding (3.35), (3.36), and (3.36) side by side and then using (3.34) we have

$$
\mathbb{E}_{x, i} \mathbf{1}_{\left\{\zeta \geq T_{2}\right\}} U\left(X\left(T_{2}\right)\right) \leq p U(x) \text {, for }(x, i) \in B_{h} \times \mathbb{Z}_{+} .
$$

By the strong Markov property of the process $(X(t), \alpha(t))$,

$$
\begin{aligned}
\mathbb{E}_{x, i} \mathbf{1}_{\left\{\zeta \geq 2 T_{2}\right\}} U\left(X\left(2 T_{2}\right)\right) & =\mathbb{E}_{x, i}\left[\mathbf{1}_{\left\{\zeta \geq T_{2}\right\}} \mathbb{E}_{X\left(T_{2}\right), \alpha\left(T_{2}\right)} \mathbf{1}_{\left\{\zeta \geq T_{2}\right\}} U\left(X\left(T_{2}\right)\right)\right] \\
& \leq p \mathbb{E}_{x, i} \mathbf{1}_{\left\{\zeta \geq T_{2}\right\}} U\left(X\left(T_{2}\right)\right) \\
& \leq p^{2} U(x), \text { for }(x, i) \in B_{h} \times \mathbb{Z}_{+} .
\end{aligned}
$$

Continuing this way we have

$$
\mathbb{E}_{x, i} \mathbf{1}_{\left\{\zeta \geq k T_{2}\right\}} U\left(X\left(k T_{2}\right)\right) \leq p^{k} U(x), \text { for }(x, i) \in B_{h} \times \mathbb{Z}_{+} .
$$


Since $2 \theta<1$, we have from (3.16) that $\mathbb{E}_{x, i} e^{2 \theta H(s)} \leq e^{2 \theta\left[\bar{c}+M_{g}\right] s}$. This and the Burkholder-DavisGundy inequality imply

$$
\begin{aligned}
\mathbb{E}_{x, i} \sup _{t \leq T_{2}}\left|\int_{0}^{t \wedge \tau_{h}} e^{\theta H(s)} \frac{V_{x}(X(s)) \sigma(X(s), \alpha(s))}{g(V(X(s)))} d W(s)\right| \\
\leq\left[\mathbb{E}_{x, i} \int_{0}^{T_{2} \wedge \tau_{h}} e^{2 \theta H(s)} \frac{\left|V_{x}(X(s)) \sigma(X(s), \alpha(s))\right|^{2}}{g^{2}(V(X(s)))} d s\right]^{\frac{1}{2}} \\
\leq\left[M_{g}^{2} \mathbb{E}_{x, i} \int_{0}^{T_{2}} e^{2 \theta H(s)} d s\right]^{\frac{1}{2}} \\
\leq\left[M_{g}^{2} \int_{0}^{T_{2}} e^{2 \theta\left[\bar{c}+M_{g}\right] s} d s\right]^{\frac{1}{2}}:=\widetilde{K}_{1} .
\end{aligned}
$$

On the other hand

$$
\begin{aligned}
\mathbb{E}_{x, i} \sup _{t \leq T_{2}}\left|\int_{0}^{t \wedge \tau_{h}} e^{\theta H(s)}\left[\theta c(\alpha(s))+\frac{\theta^{2}}{2} \frac{\left|V_{x}(X(s)) \sigma(X(s), \alpha(s))\right|^{2}}{2 g^{2}(V(X(s)))}\right] d s\right| \\
\quad \leq\left(\bar{c}+M_{g}\right) \mathbb{E}_{x, i} \int_{0}^{T_{2} \wedge \tau_{h}} e^{\theta H(s)} d s \\
\quad \leq\left(\bar{c}+M_{g}\right) \int_{0}^{T_{2}} e^{\theta\left[\bar{c}+M_{g}\right]} d s:=\widetilde{K}_{2} .
\end{aligned}
$$

It follows from (3.38) and (3.39) that

$$
\begin{aligned}
\mathbb{E}_{x, i} \sup _{t \leq T_{2}} U\left(X\left(t \wedge \tau_{h}\right)\right) & =U(x) \mathbb{E}_{x, i} \sup _{t \leq T_{2}} e^{\theta H_{t}} \\
& \leq U(x)\left[1+\widetilde{K}_{1}+\widetilde{K}_{2}\right]:=U(x) \widetilde{K}_{3} .
\end{aligned}
$$

By the strong Markov property of $(X(t), \alpha(t))$, we derive from (3.40) that

$$
\begin{aligned}
\mathbb{E}_{x, i} \mathbf{1}_{\{\zeta=\infty\}} \sup _{t \in\left[k T_{2},(k+1) T_{2}\right]} U\left(X\left(t \wedge \tau_{h}\right)\right) \\
\quad \leq \mathbb{E}_{x, i} \mathbf{1}_{\left\{\zeta \geq k T_{2}\right\}} \sup _{t \in\left[k T_{2},(k+1) T_{2}\right]} U\left(X\left(t \wedge \tau_{h}\right)\right) \\
\leq \widetilde{K}_{3} \mathbb{E}_{x, i} \mathbf{1}_{\left\{\zeta \geq k T_{2}\right\}} U\left(X\left(k T_{2}\right)\right. \\
\leq \widetilde{K}_{3} U(x) \rho^{k},
\end{aligned}
$$

which combined with Markov's inequality leads to

$$
\begin{aligned}
\mathbb{P}_{x, i} & \left\{\mathbf{1}_{\{\zeta=\infty\}} \sup _{t \in\left[k T_{2},(k+1) T_{2}\right]} U\left(X\left(t \wedge \tau_{h}\right)\right)>(\rho+\widetilde{\varepsilon})^{k}\right\} \\
& \leq \frac{1}{(\rho+\widetilde{\varepsilon})^{k}} \mathbb{E}_{x, i}\left[\mathbf{1}_{\{\zeta=\infty\}} \sup _{t \in\left[k T_{2},(k+1) T_{2}\right]} U\left(X\left(t \wedge \tau_{h}\right)\right)\right] \\
& \leq \widetilde{K}_{3} U(x) \frac{\rho^{k}}{(\rho+\widetilde{\varepsilon})^{k}} \quad k \in \mathbb{Z}_{+},
\end{aligned}
$$

where $\widetilde{\varepsilon}$ is any number in $(0,1-\rho)$. In view of the Borel-Cantelli lemma, for almost all $\omega \in \Omega$, there exists random integer $k_{1}=k_{1}(\omega)$ such that

$$
\mathbf{1}_{\{\zeta=\infty\}} \sup _{t \in\left[k T_{2},(k+1) T_{2}\right]} U(X(t))<(\rho+\widetilde{\varepsilon})^{k} \text { for any } k \geq k_{1} .
$$


Thus, for almost all $\omega \in\{\zeta=\infty\}$, we have

$$
G(V(X(t))) \leq\left[t / T_{2}\right] \ln (\rho+\widetilde{\varepsilon}) \leq-\lambda t \text { for } t \geq k_{1} T_{2} .
$$

where $\left[t / T_{2}\right]$ is the integer part of $t / T_{2}$ and $\lambda=-\frac{\ln (\rho+\widetilde{\varepsilon})}{2 T_{2}}>0$. Since $G(y)$ is decreasing and maps $(0, h]$ onto $(-\infty, 0],(3.12)$ follows from (3.33) and (3.43)

In Theorem 3.2, under the condition that $\alpha(t)$ is merely ergodic, we need and additional condition (3.10) to obtain the stability in probability of the system. If $\alpha(t)$ is strongly ergodic, the condition (3.10) can be removed.

Theorem 3.3. Suppose that

- for any $T>0$ and a bounded function $f: \mathbb{Z}_{+} \mapsto \mathbb{R}$, we have

$$
\lim _{x \rightarrow 0} \sup _{i \in \mathbb{Z}_{+}}\left\{\mid \mathbb{E}_{x, i} \int_{0}^{T} f(\alpha(s)) d s-\mathbb{E}_{i} \int_{0}^{T} f(\widehat{\alpha}(s) d s \mid\}=0 .\right.
$$

- Assumption 3.1 is satisfied

- the Markov chain $\widehat{\alpha}(t)$ is strongly ergodic with invariant probability measure $\boldsymbol{\nu}=\left(\nu_{1}, \nu_{2}, \ldots\right)$. Suppose further that (3.11) is satisfied and $\sum_{i \in \mathbb{Z}_{+}} c_{i} \nu_{i}<0$. Then the conclusion of Theorem 3.2 holds.

Remark 3.2. We will prove in the Appendix that (3.44) holds if Assumption 3.1 and (3.1) hold.

Proof of Theorem 3.3. Let $\lambda=-\sum_{i \in \mathbb{Z}_{+}} c_{i} \nu_{i}$. Because of the uniform ergodicity of $\widehat{\alpha}(t)$, there exists a $T>0$ such that

$$
\mathbb{E}_{0, i} \int_{0}^{t} c(\alpha(s)) d s=\mathbb{E}_{i} \int_{0}^{t} c(\widehat{\alpha}(s)) d s \leq-\frac{3 \lambda}{4} t \quad \forall t \geq T, i \in \mathbb{Z}_{+} .
$$

By (3.44), there exists $h_{1} \in(0, h)$ such that

$$
\mathbb{E}_{x, i} \int_{0}^{T} c(\alpha(s)) d s \leq-\frac{\lambda}{2} T \quad \forall|x| \leq h_{1}, i \in \mathbb{Z}_{+} .
$$

In view of Lemma 3.1, there exists $h_{2} \in\left(0, h_{1}\right)$ such that

$$
\bar{c} \mathbb{P}_{x, i}\left\{\tau_{h}<T\right\} \leq \frac{\lambda}{4} \text { provided }|x| \leq h_{2}, i \in \mathbb{Z}_{+}
$$

Applying (3.46) and (3.47) to (3.17), we have

$$
\mathbb{E}_{x, i} H(T) \leq-\frac{\lambda}{4} T \text { if } 0<|x| \leq h_{2}, i \in \mathbb{Z}_{+} .
$$

Using (3.48), we can use arguments in the proof Theorem 3.2 to show that

$$
\mathbb{E}_{x, i} e^{\theta H(T)} \leq \exp \left\{-\frac{\theta \lambda T}{8}\right\} \text { for } 0<|x|<h_{2}
$$


for a sufficiently small $\theta>0$. This implies that

$$
\mathbb{E}_{x, i} U\left(X\left(T \wedge \tau_{h}\right)\right) \leq \exp \left\{-\frac{\theta \lambda T}{8}\right\} U(x)
$$

where $U(x)=\exp (\theta G(V(x)))$. Thus, $\left\{M_{k}:=U\left(X\left((k T) \wedge \tau_{h}\right)\right), k=0,1, \ldots\right\}$ is a bounded supermartingale. Then we can easily obtain the stability in probability of the trivial solution. Moreover, proceeding as in Step 2 of the proof of Theorem 3.2, we can obtain the asymptotic stability as well as the rate of convergence. The arguments are actually simpler because (3.50) holds uniformly in $i \in \mathbb{Z}_{+}$, rather than $i \in\left\{1, \ldots, k_{0}\right\}$ in the proof of Theorem 3.2 .

Remark 3.3. Consider the special case $g(y) \equiv y$. With this function we have $U(X(t))=V(X(t))$. Thus, if Assumption 3.1 holds with $g(y) \equiv y$, then the conclusion on stability in Theorems 3.2 and 3.3 are still true without the condition (3.11) because we still have $\mathbb{E} V\left(X\left(t \wedge \tau_{h}\right)\right) \leq V(x) e^{\bar{c} t}$, which can be used in place of (3.27). However, in order to obtain asymptotic stability and rate of convergence, (3.11) is needed. In that case, if the initial value is sufficiently closed to $0, V(X(t))$ will converges exponentially fast to 0 with a large probability.

Theorem 3.4. Consider the case that the state space of $\alpha(t)$ is finite, say $\mathcal{M}=\left\{1, \ldots, m_{0}\right\}$ for some positive integer $m_{0}$, rather than $\mathbb{Z}_{+}$. Suppose that $Q(0)$ is irreducible and let $\boldsymbol{\nu}$ be the invariant probability measure of the Markov chain with generator $Q(0)$. If $\sum_{i \in \mathcal{M}} c_{i} \nu_{i}<0$ then the trivial solution is asymptotically stable in probability, and for any $\varepsilon>0$, there are $\lambda>0, \delta>0$ such that

$$
\mathbb{P}_{x, i}\left\{\lim _{t \rightarrow \infty} \frac{V(X(t))}{G^{-1}\left(-\lambda_{3} t\right)} \leq 1\right\}>1-\varepsilon \text { for any }(x, i) \in B_{\delta} \times \mathbb{Z}_{+} .
$$

We now provide some conditions for instability in probability.

Theorem 3.5. Suppose that the Markov chain $\widehat{\alpha}(t)$ is ergodic with invariant probability measure $\boldsymbol{\nu}=\left(\nu_{1}, \nu_{2}, \ldots\right)$ and that there are functions $g \in \Gamma, V: D \mapsto \mathbb{R}_{+}$such that

- $V(x)=0$ if and only if $x=0$

- $V(x)$ is continuous on $D$ and twice continuously differentiable in $D \backslash\{0\}$.

- there is a bounded sequence of real numbers $\left\{c_{i}: i \in \mathbb{Z}_{+}\right\}$such that

$$
\mathcal{L}_{i} V(x) \geq c_{i} g(V(x)) \forall x \in D \backslash\{0\} .
$$

If (3.11) is satisfied and if $\sum_{i \in \mathbb{Z}_{+}} c_{i} \nu_{i}<0$ and $\limsup _{i \rightarrow \infty} c_{i}<0$, then the trivial solution is unstable in probability.

Proof. Define $G(y)=-\int_{y}^{h} g^{-1}(z) d z$ as in Theorem 3.2. We have from Itô's formula,

$$
\begin{aligned}
-G\left(V\left(X\left(\tau_{h} \wedge t\right)\right)\right)= & -G(V(x))-\int_{0}^{\tau_{h} \wedge t} \frac{\mathcal{L}_{\alpha(s)} V(X(s))}{g(V(X(s)))} d s \\
& +\int_{0}^{\tau_{h} \wedge t} \frac{\dot{g}(V(X(s)))\left|V_{x}(X(s)) \sigma(X(s), \alpha(s))\right|^{2}}{2 g^{2}(V(X(s)))} d s \\
& -\int_{0}^{\tau_{h} \wedge t} \frac{V_{x}(X(s)) \sigma(X(s), \alpha(s))}{g(V(X(s)))} d W(s) \leq-G(V(x))+\widetilde{H}(t)
\end{aligned}
$$


where

$$
\widetilde{H}(t)=-\int_{0}^{\tau_{h} \wedge t} c(\alpha(s)) d s-\int_{0}^{\tau_{h} \wedge t} \frac{V_{x}(X(s)) \sigma(X(s), \alpha(s))}{g(V(X(s)))} d W(s) .
$$

Then using (3.52) and proceeding in the same manner as in the proof of Theorem 3.2 with $H(t)$ replaced with $\widetilde{H}(t)$, we have can find a sufficiently small $\widetilde{\theta}, \widetilde{\Delta}>0$ and a sufficiently large $T_{3}>0$ such that

$$
\mathbb{E}_{x, i} \mathbf{1}_{\left\{\widetilde{\zeta} \geq k T_{3}\right\}} \widetilde{U}\left(X\left(k T_{2}\right)\right) \leq p^{k} \widetilde{U}(x), \text { for }(x, i) \in B_{h} \times \mathbb{Z}_{+} .
$$

where $\widetilde{U}(x)=\exp \{-\tilde{\theta} G(V(x))\}$, and $\widetilde{\zeta}=\inf \left\{k \geq 0: U\left(X\left(k T_{3}\right)\right) \leq \widetilde{\Delta}^{-1}\right\}$. Note that, unlike $U(x)$, we have $\lim _{x \rightarrow 0} \widetilde{U}(x)=\infty$. Since $U\left(X\left(k T_{3}\right)\right) \geq \widetilde{\Delta}^{-1}$ if $\widetilde{\zeta} \geq k$, we have that

$$
\mathbb{P}_{x, i}\{\widetilde{\zeta}=\infty\}=\lim _{k \rightarrow \infty} \mathbb{P}_{x, i}\{\widetilde{\zeta} \geq k\}=0 .
$$

Similarly, we can obtain a counterpart of Theorem 3.3 for instability.

Theorem 3.6. Suppose that the Markov chain $\widehat{\alpha}(t)$ is strongly ergodic with invariant probability measure $\boldsymbol{\nu}=\left(\nu_{1}, \nu_{2}, \ldots\right)$ and that there are functions $g \in \Gamma, V: D \mapsto \mathbb{R}_{+}$such that

- $V(x)=0$ if and only if $x=0$

- $V(x)$ is continuous on $D$ and twice continuously differentiable in $D \backslash\{0\}$.

- there is a bounded sequence of real numbers $\left\{c_{i}: i \in \mathbb{Z}_{+}\right\}$such that

$$
\mathcal{L}_{i} V(x) \geq c_{i} g(V(x)) \forall x \in D \backslash\{0\} .
$$

If (3.11) and (3.1) are satisfied and if $\sum_{i \in \mathbb{Z}_{+}} c_{i} \nu_{i}>0$ then the trivial solution is unstable in probability.

\section{$4 \quad$ Linearized Systems}

Suppose that (3.44) is satisfied and that $\widehat{\alpha}(t)$ is a strongly ergodic Markov chain.

Assumption 4.1. Suppose that for $i \in \mathbb{Z}_{+}$, there exist $b(i), \sigma_{k}(i) \in \mathbb{R}^{n \times n}$ bounded uniformly for $i \in \mathbb{Z}_{+}$such that

$$
\xi_{i}(x):=b(x, i)-b(i) x, \quad \zeta_{i}(x):=\sigma(x, i)-\left(\sigma_{1}(i) x, \ldots, \sigma_{d}(i) x\right)
$$

satisfying

$$
\lim _{x \rightarrow 0} \sup _{i \in \mathbb{Z}_{+}}\left\{\frac{\left|\xi_{i}(x)\right| \vee\left|\zeta_{i}(x)\right|}{|x|}\right\}=0
$$

For $i \in \mathbb{Z}_{+}, k \in\{1, \ldots, n\}$, let $\Lambda_{1, i}$ and $\Lambda_{2, i, k}$ be the maximum eigenvalues of $\frac{b(i)+b^{\top}(i)}{2}$ and $\sigma_{k}(i) \sigma_{k}^{\top}(i)$ respectively. Similarly, denote by $\lambda_{1, i}$ and $\lambda_{2, i, k}$ be the minimum eigenvalues of $\frac{b(i)+b^{\top}(i)}{2}$ and $\sigma_{k}(i) \sigma_{k}^{\top}(i)$ respectively. 
Suppose that $\Lambda_{1, i}$ and $\Lambda_{2, i, k}$ are bounded in $i \in \mathbb{Z}_{+}$then we claim that if

$$
\sum_{i \in \mathbb{Z}_{+}} \nu_{i}\left(\Lambda_{1, i}+\frac{1}{2} \sum_{k=1}^{n} \Lambda_{2, i, k}\right)<0
$$

then the trivial solution is asymptotic stable.

To show that, let $\varepsilon>0$ be sufficiently small such that

$$
\sum_{i \in \mathbb{Z}_{+}} \nu_{i}\left(\varepsilon+\Lambda_{1, i}+\frac{1}{2} \sum_{k=1}^{n} \Lambda_{2, i, k}\right)<0 .
$$

Define $V(x)=|x|^{p}$, carry out the calculation and obtain the estimates as in that of [7, Theorem 4.3], we can find a sufficiently small $p>0$ and $\hbar>0$ such that

$$
\mathcal{L}_{i} V(x) \leq p\left(\varepsilon+\Lambda_{1, i}+\frac{1}{2} \sum_{k=1}^{n} \Lambda_{2, i, k}\right) V(x) \text { for } 0<|x|<\hbar .
$$

(Note that the existence of such $p$ and $\hbar$ satisfying (4.3) uniformly for $i \in \mathbb{Z}_{+}$is due to (4.3) and the boundedness of $\Lambda_{1, i}$ and $\Lambda_{2, i, k}$.)

By (4.2) and (4.3), it follows from Theorem 3.3 that the trivial solution is asymptotic stable and for any $\varepsilon>0$, there exists $\delta>0, \lambda>0$ such that

$$
\mathbb{P}_{x, i}\left\{\lim _{t \rightarrow \infty} e^{\lambda t}|X(t)| \leq 1\right\} \geq 1-\varepsilon \text { for }(x, i) \in B_{\delta} \times \mathbb{Z}_{+} .
$$

Similarly, if $\sum_{i \in \mathbb{Z}_{+}} \nu_{i}\left(\lambda_{1, i}+\frac{1}{2} \sum_{k=1}^{n} \lambda_{2, i, k}\right)>0$, and if $\lambda_{1, i}$ and $\lambda_{2, i, k}$ are bounded in $i \in$ $\mathbb{Z}_{+}, k=1, \ldots, n$, we have that the trivial solution is unstable. To sum up, we have the following result.

Proposition 4.1. Let Assumption 4.1 is satisfied. We claim that,

- if $\Lambda_{1, i}$ and $\Lambda_{2, i, k}$ are bounded in $i \in \mathbb{Z}_{+}$and

$$
\sum_{i \in \mathbb{Z}_{+}} \nu_{i}\left(\Lambda_{1, i}+\frac{1}{2} \sum_{k=1}^{n} \Lambda_{2, i, k}\right)<0
$$

then the trivial solution is asymptotically stable in probability;

- if $\lambda_{1, i}$ and $\lambda_{2, i, k}$ are bounded in $i \in \mathbb{Z}_{+}, k=1, \ldots, n$, and

$$
\sum_{i \in \mathbb{Z}_{+}} \nu_{i}\left(\lambda_{1, i}+\frac{1}{2} \sum_{k=1}^{n} \lambda_{2, i, k}\right)>0
$$

then the trivial solution is unstable in probability. 


\section{$5 \quad$ Examples}

This section provides several examples.

Example 5.1. Consider a real-valued switching diffusion

$$
d X(t)=b(\alpha(t)) X(t)\left[|X(t)|^{\gamma} \vee 1\right] d t+\sigma(\alpha(t)) \sin ^{2} X(t) d W(t), 0<\gamma<1,
$$

where $a \vee b=\max (a, b)$ for two real numbers $a$ and $b$, and $Q(x)=\left(q_{i j}(x)\right)_{\mathbb{Z}_{+} \times \mathbb{Z}_{+}}$with

$$
q_{i j}(x)= \begin{cases}-\check{p}_{1}(x) & \text { if } i=j=1 \\ \check{p}_{1}(x) & \text { if } i=1, j=2 \\ -\widehat{p}_{i}(x)-\check{p}_{i}(x) & \text { if } i=j \geq 2 \\ \widehat{p}_{i}(x) & \text { if } i \geq 2, j=i-1 \\ \check{p}_{i}(x) & \text { if } i \geq 2, j=i+1 .\end{cases}
$$

Note that the drift grow faster than linear and the diffusion coefficient is locally like $x^{2}$ near the origin for the continuous state. Suppose that $b(i), \sigma(i), \check{p}_{i}(x), \widehat{p}_{i}(x)$ are bounded for $(x, i) \in \mathbb{R} \times \mathbb{Z}_{+}$ and $\check{p}_{i}(x), \widehat{p}_{i}(x)$ are continuous in $\mathbb{R}^{n}$ for each $i \in \mathbb{Z}_{+}$. It is well known (see [1, Chapter 8]) that if

$$
\nu^{*}:=\sum_{k=2}^{\infty} \prod_{\ell=2}^{k} \frac{\check{p}_{\ell-1}(0)}{\widehat{p}_{\ell}(0)}<\infty,
$$

then $\widehat{\alpha}(t)$ is ergodic with the invariant measure $\boldsymbol{\nu}$ given by

$$
\nu_{1}=\frac{1}{\nu^{*}}, \nu_{k}=\frac{1}{\nu^{*}} \prod_{\ell=2}^{k} \frac{\check{p}_{\ell-1}(0)}{\widehat{p}_{\ell}(0)}, k \geq 2 .
$$

We suppose that

$$
\sum b(i) \nu_{i}<0, \text { and } \limsup _{i \rightarrow \infty} b(i)<0 .
$$

we will show that the trivial solution is stable. Let $0<\varepsilon<-\sum b(i) \nu_{i}$ then $\sum[b(i)+\varepsilon] \nu_{i}<0$. Let

$$
V(x)=x^{2}
$$

We have

$$
\mathcal{L}_{i} V(x)=2 b(i)|x|^{2+2 \gamma}+\sigma^{2}(i) \sin ^{4}(x)
$$

Since $\gamma<1$ and $\sigma(i)$ is bounded, there exists an $\hbar>0$ such that $\sigma^{2}(i) \sin ^{4}(x) \leq \varepsilon|x|^{2+2 \gamma}$ given that $|x| \leq \hbar$. then

$$
\mathcal{L}_{i} V(x) \leq[2 b(i)+\varepsilon]|x|^{2+2 \gamma}=[2 b(i)+\varepsilon] V^{1+\gamma}(x) \text { in }[-\hbar, \hbar] \times \mathbb{Z}_{+} .
$$

By Theorem 3.2, the trivial solution is asymptotically stable in probability. Moreover, for the function $g(y)=y^{1+\gamma}$,

$$
G(y):=-\int_{y}^{\hbar} \frac{1}{g(z)} d s=\hbar^{-\gamma}-y^{-\gamma}, y \in(0, \hbar]
$$

has the inverse

$$
G^{-1}(-t)=\frac{1}{\left[t+\hbar^{-\gamma}\right]^{1 / \gamma}}, \text { for } t \geq 0
$$

Thus, for any $\varepsilon>0$, there exists a $\delta>0$ such that if $(x, i) \in[0, \delta] \times \mathbb{Z}_{+}$, then, there exists a $\lambda>0$ such that

$$
\mathbb{P}_{x, i}\left\{\limsup _{t \rightarrow \infty} t^{1 / \gamma} X^{2}(t) \leq \lambda\right\}>1-\varepsilon .
$$


Example 5.2. This example consider a random-switching linear systems of differential equations:

$$
d X(t)=A(\alpha(t)) X(t) d t
$$

where $A(i) \in \mathbb{R}^{n \times n}$ satisfying $\sup _{i \in \mathbb{Z}_{+}}\left\{\left|\lambda_{i}\right| \vee\left|\Lambda_{i}\right|\right\}<\infty$ with $\lambda_{i}, \Lambda_{i}$ being the minimum and maximum eigenvalues of $A(i)$, respectively. Let

$$
Q(x)=\left(\begin{array}{cccccc}
-1-\sin |x| & 1+\sin |x| & 0 & 0 & 0 & \cdots \\
1+\sin |x| & -2-2 \sin |x| & 1+\sin |x| & 0 & 0 & \cdots \\
1+\sin |x| & 0 & -2-2 \sin |x| & 1+\sin |x| & 0 & \cdots \\
1+\sin |x| & 0 & 0 & -2-2 \sin |x| & 1+\sin |x| & \cdots \\
\vdots & \vdots & \vdots & \vdots & \vdots & \ddots
\end{array}\right) .
$$

By [1, Proposition 3.3], it is easy to verify that the Markov chain $\widehat{\alpha}(t)$ with generators $Q(0)$ is strongly ergodic. Solving the system

$$
\boldsymbol{\nu} Q(0)=0, \sum \boldsymbol{\nu}_{i}=1
$$

we obtain that the invariant measure of $\widehat{\alpha}(t)$ is $\left(\nu_{i}\right)_{i=1}^{\infty}=\left(2^{-i}\right)_{i=1}^{\infty}$. Thus, if $\sum \lambda_{i} 2^{-i}>0$ the trivial solution to (5.2) is unstable. In case $\sum \Lambda_{i} 2^{-i}<0$ the trivial solution to (5.2) is asymptotically stable in probability. In particular, suppose that $n=2$ and $A(i)$ are upper triangle matrices, that is,

$$
A(i)=\left(\begin{array}{cc}
a_{i} & b_{i} \\
0 & c_{i}
\end{array}\right)
$$

If $a_{i}$ and $c_{i}$ are positive for $i \geq 2$, then the system $d X(t)=A(i) X(t) d t$ is unstable. However, if $a_{1}, c_{1}<-\sup _{i \geq 2}\left\{a_{i}, c_{i}\right\}$, then $\sum\left(a_{i} \vee c_{i}\right) 2^{-i}<0$. Thus, the switching differential system is asymptotically stable. The stability of the system at state 1 and the switching process become a stabilizing factor.

On the other hand, if $a_{i} \wedge c_{i}$ is negative for $i \geq 2$, then the system $d X(t)=A(i) X(t) d t$ is asymptotically stable. Suppose further that $a_{1}, c_{1}>\sup _{i \geq 2}\left\{-\left(a_{i} \wedge c_{i}\right)\right\}$, then $\sum\left(a_{i} \vee c_{i}\right) 2^{-i}>0$. Under this condition, the switching differential system is unstable.

\section{$6 \quad$ Further Remarks}

Using a new method, we provide sufficient conditions for stability and instability in probability of a class of regime-switching diffusion systems with switching states belonging to a countable set. The conditions are based on the relation of a "switching-independent" Lyapunov function and the generator of the switching part.

Although the systems under consideration are memoryless, the main results of this paper hold if we assume that the switching intensities $q_{i j}$ depend on the history of $\{X(t)\}$ rather than the current state of $X(t)$, (see [11, 12] for fundamental properties of this process). The problem can be formulated as follows. Let $r$ be a fixed positive number. Denote by $\mathcal{C}$ the set of $\mathbb{R}^{n}$-valued continuous functions defined on $[-r, 0]$. For $\phi \in \mathcal{C}$, we use the norm $\|\phi\|=\sup \{|\phi(t)|: t \in[-r, 0]\}$. For $t \geq 0$, we denote by $y_{t}$ the so-called segment function (or memory segment function) $y_{t}=$ $\{y(t+s):-r \leq s \leq 0\}$. We assume that the jump intensity of $\alpha(t)$ depends on the trajectory of $X(t)$ in the interval $[t-r, t]$. That is, there are functions $q_{i j}(\cdot): \mathcal{C} \rightarrow \mathbb{R}$ for $i, j \in \mathbb{Z}_{+}$satisfying 
that $q_{i}(\phi):=\sum_{j=1, j \neq i}^{\infty} q_{i j}(\phi)$ is uniformly bounded in $(\phi, i) \in \mathcal{C} \times \mathbb{Z}_{+}$and that $q_{i}(\cdot)$ and $q_{i j}(\cdot)$ are continuous such that

$$
\begin{aligned}
& \mathbb{P}\left\{\alpha(t+\Delta)=j \mid \alpha(t)=i, X_{s}, \alpha(s), s \leq t\right\}=q_{i j}\left(X_{t}\right) \Delta+o(\Delta) \text { if } i \neq j \text { and } \\
& \mathbb{P}\left\{\alpha(t+\Delta)=i \mid \alpha(t)=i, X_{s}, \alpha(s), s \leq t\right\}=1-q_{i}\left(X_{t}\right) \Delta+o(\Delta) .
\end{aligned}
$$

It is proved in [11] that if either Assumption 4.1 or Assumption 2.2 is satisfied with $x, y \in \mathbb{R}^{n}$ replaced by $\phi, \psi \in \mathcal{C}$, then there is a unique solution to the switching diffusion (2.1) and (6.1) with a given initial value. Moreover, the process $\left(X_{t}, \alpha(t)\right)$ has the Markov-Feller property. With slight modification in the proofs, the theorems in Section 3 still hold for system (2.1) and (6.1).

Our method can also be applied to regime-switching jump diffusion processes. The results obtain by using our method will generalize existing results (e.g., [15, 18]) to the case of regime-switching jump diffusions with countable regimes.

On the other hand, there is a gap between sufficient conditions for stability and instability in Proposition 4.1. To overcome the difficulty, we need to make a polar coordinate transformation to decompose of $X(t)$ into the radial part $r(t)=|X(t)|$ and the angular part $Y(t)=X(t) / r(t)$. Then, the Lyapunov exponents with respect to invariant measures of the linearized process of $(Y(t), \alpha(t))$ will determine whether or not the system is stable. This approach has been used to treat many linear and linearized stochastic systems (e.g., [2, 3, 6]). In our setting, the switching $\alpha(t)$ take values in a noncompact space, thus, it is more difficult to examine invariant measures. We will address this problem together necessary conditions of stability in a subsequent paper.

\section{A Appendix}

Proof of Lemma 3.1. Since $g$ is continuously differentiable and $g(0)=0$, there is $K_{g}>0$ such that $g(z) \leq K_{g}|z|$ for $|z| \leq 1$. Thus, we have

$$
\mathcal{L}_{i} V(x) \leq K_{g} \sup _{i \in \mathbb{Z}_{+}}\left\{\left|c_{i}\right|\right\} V(x),(x, i) \in D \times \mathbb{Z}_{+}
$$

Letting $\tilde{K}=K_{g} \sup _{i \in \mathbb{Z}_{+}}\left\{\left|c_{i}\right|\right\}$, by Itô's formula,

$$
\begin{aligned}
\mathbb{E}_{x, i} V\left(X\left(t \wedge \tau_{h}\right)\right) & \leq E V(x)+\tilde{K} \mathbb{E}_{x, i} \int_{0}^{t \wedge \tau_{h}} V(X(s)) d s \\
& \leq E V(x)+\tilde{K} \mathbb{E}_{x, i} \int_{0}^{t} \mathbb{E}_{x, i} V\left(X\left(s \wedge \tau_{h}\right)\right) d s .
\end{aligned}
$$

By the Grownwall inequality, we can easily obtain

$$
\mathbb{E}_{x, i} V\left(X\left(T \wedge \tau_{h}\right)\right) \leq V(x) e^{K T} .
$$

Since $V(0)=0$, standard arguments lead to the desired result.

Proof of Lemma 3.2. It is easy to show that there exists some $K_{2}>0$ such that

$$
|y|^{k} \exp (\theta y) \leq K_{2}\left(\exp \left(\theta_{0} y\right)+\exp \left(-\theta_{0} y\right)\right), k=1,2 .
$$

for $\theta \in\left[0, \frac{\theta_{0}}{2}\right], y \in \mathbb{R}$. For any $y \in \mathbb{R}$, let $\xi(y)$ be a number lying between $y$ and 0 such that $\exp (\xi(y))=\frac{e^{y}-1}{y}$. Pick $\theta \in\left[0, \frac{\theta_{0}}{2}\right]$ and let $h \in \mathbb{R}$ such that $0 \leq \theta+h \leq \frac{\theta_{0}}{2}$. Then

$$
\lim _{h \rightarrow 0} \frac{\exp ((\theta+h) Y)-\exp (\theta Y)}{h}=Y \exp (\theta Y) \text { a.s. }
$$


where $Y$ is as defined in Lemma 3.2, and

$$
\left|\frac{\exp ((\theta+h) Y)-\exp (\theta Y)}{h}\right|=|Y| \exp (\theta Y+\xi(h Y)) \leq 2 K_{3}\left[\exp \left(\theta_{0} Y\right)+\exp \left(-\theta_{0} Y\right)\right] .
$$

By the Lebesgue dominated convergence theorem,

$$
\frac{d \mathbb{E} \exp (\theta Y)}{d \theta}=\lim _{h \rightarrow 0} \mathbb{E} \frac{\exp ((\theta+h) Y)-\exp (\theta Y)}{h}=\mathbb{E} Y \exp (\theta Y) .
$$

Similarly,

$$
\frac{d^{2} \mathbb{E} \exp (\theta Y)}{d \theta^{2}}=\mathbb{E} Y^{2} \exp (\theta Y)
$$

As a result, we obtain

$$
\frac{d \phi}{d \theta}=\frac{\mathbb{E} Y \exp (\theta Y)}{\mathbb{E} \exp (\theta Y)}
$$

which implies

$$
\frac{d \phi}{d \theta}(0)=\mathbb{E} Y
$$

and

$$
\frac{d^{2} \phi}{d \theta^{2}}=\frac{\mathbb{E} Y^{2} \exp (\theta Y) \mathbb{E} \exp (\theta Y)-[\mathbb{E} Y \exp (\theta Y)]^{2}}{[\mathbb{E} \exp (\theta Y)]^{2}}
$$

By Hölder's inequality we have $\mathbb{E} Y^{2} \exp (\theta Y) \mathbb{E} \exp (\theta Y) \geq[\mathbb{E} Y \exp (\theta Y)]^{2}$ and therefore

$$
\frac{d^{2} \phi}{d \theta^{2}} \geq 0, \forall \theta \in\left[0, \frac{\theta_{0}}{2}\right] \text {. }
$$

Moreover,

$$
\begin{aligned}
\frac{d^{2} \phi}{d \theta^{2}} & \leq \frac{\mathbb{E} Y^{2} \exp (\theta Y)}{\mathbb{E} \exp (\theta Y)} \\
& \leq \frac{K_{3}\left(\mathbb{E} \exp \left(\theta_{0} Y\right)+\mathbb{E} \exp \left(-\theta_{0} Y\right)\right)}{\exp (\theta \mathbb{E} Y)} \\
& \leq \frac{K_{3}\left(\mathbb{E} \exp \left(\theta_{0} Y\right)+\mathbb{E} \exp \left(-\theta_{0} Y\right)\right)}{\exp \left(-\theta_{0}|\mathbb{E} Y|\right)}:=K_{2},
\end{aligned}
$$

which concludes the proof.

Proof of Lemma 3.3. Let $\bar{\tau}_{n}$ be the $n$-th jump moment of $\alpha(t)$. Let $T>0$, In view of [9, Lemma 4.3.2], we have

$$
\mathbb{P}_{x, i}\left\{X(t)=0 \text { for some } t \in\left[0, T \wedge \bar{\tau}_{1}\right]\right\}=0 \text { for any } x \neq 0, i \in \mathbb{Z}_{+} .
$$

Since $X\left(T \wedge \bar{\tau}_{1}\right) \neq 0$ a.s., applying [9, Lemma 4.3.2] again yields

$$
\mathbb{P}_{x, i}\left\{X(t)=0 \text { for some } t \in\left[T \wedge \bar{\tau}_{1}, T \wedge \bar{\tau}_{2}\right]\right\}=0 \text { for any } x \neq 0, i \in \mathbb{Z}_{+} .
$$

Continuing this way, we have

$$
\mathbb{P}_{x, i}\left\{X(t)=0 \text { for some } t \in\left[0, T \wedge \bar{\tau}_{n}\right]\right\}=0 \text { for any } x \neq 0, i \in \mathbb{Z}_{+}, n \in \mathbb{Z}_{+} .
$$

In [11, Theorems $3.1 \& 3.3$ ], we have that $\lim _{n \rightarrow \infty} \bar{\tau}_{n}=\infty$. This and (A.1) imply

$$
\mathbb{P}_{x, i}\{X(t)=0 \text { for some } t \in[0, T]\}=0 \text { for any } x \neq 0, i \in \mathbb{Z}_{+}, n \in \mathbb{Z}_{+} .
$$

Since $T$ is taken arbitrarily, we obtain the desired result. 
Lemma A.1. If the Markov chain $\widehat{\alpha}(t)$ is strongly exponentially ergodic with generator $\widehat{Q}$ and invariant probability measure $\boldsymbol{\nu}=\left(\nu_{1}, \nu_{2}, \ldots\right)^{\top}$, then if $\mathbf{b}=\left(b_{1}, b_{2}, \ldots\right)^{\top}$ is bounded satisfying $\sum \nu_{i} b_{i}=0$, then, there exists a bounded vector $\mathbf{c}=\left(c_{1}, c_{2}, \ldots\right)^{\top}$ such that $b_{i}=\sum \widehat{q}_{j i} c_{j}$.

Proof. Let $\widehat{P}(t)=\widehat{p}_{i j}(t)$, where $\widehat{p}_{i j}(t)=\mathbb{P}\{\widehat{\alpha}(t)=j \mid \alpha(0)=i\}$, the transition matrix of $\widehat{\alpha}(t)$. Let $\mathbf{c}=\left(c_{1}, c_{2}, \ldots\right)^{\top}$ where $c_{i}=\int_{0}^{\infty}\left[\nu_{j} b_{j}-\widehat{P}_{i j}(t) b_{i}\right] d t$. In view of (2.6), it is easy to see that $\mathbf{c}$ is bounded. Let $\mathbb{1}=(1,1, \ldots)$. We have

$$
\begin{aligned}
\widehat{Q} \mathbf{c} & =\int_{0}^{\infty}[\widehat{Q} \boldsymbol{\nu} \mathbb{1} \mathbf{b}-\widehat{Q} \widehat{P}(t) \mathbf{b}] d t \\
& =-\int_{0}^{\infty} \widehat{Q} \widehat{P}(t) \mathbf{b} d t \\
& =-\int_{0}^{\infty} \widehat{P}(t) \mathbf{b} d t=-\left.\widehat{P}(t) \mathbf{b}\right|_{0} ^{\infty} \\
& =-\mathbb{1} \boldsymbol{\nu} \mathbf{b}+\mathbf{b}=\mathbf{b} .
\end{aligned}
$$

Lemma A.2. Suppose that Assumption 3.1 and (3.1) hold. Then for any $T>0$ and a bounded function $f: \mathbb{Z}_{+} \mapsto \mathbb{R}$, we have

$$
\lim _{x \rightarrow 0} \sup _{i \in \mathbb{Z}_{+}, t \in[0, T]}\left\{\mid \mathbb{E}_{x, i} f(\alpha(t))-\mathbb{E}_{i} f(\widehat{\alpha}(t) \mid\}=0 .\right.
$$

Proof. By the basic coupling method (see e.g., [5, p. 11]), we can consider the joint process $(X(t), \alpha(t), \widehat{\alpha}(t))$ as a switching diffusion where the diffusion $X(t) \in \mathbb{R}^{n}$ satisfies satisfying

$$
d X(t)=b(X(t), \alpha(t)) d t+\sigma(X(t), \alpha(t)) d w(t)
$$

and the switching part $(\alpha(t), \widehat{\alpha}(t)) \in \mathbb{Z}_{+} \times \mathbb{Z}_{+}$has the generator $\widetilde{Q}(X(t))$ which is defined by

$$
\begin{aligned}
\widetilde{Q}(x) \widetilde{f}(k, l)= & \sum_{j, i \in \mathbb{Z}_{+}} \widetilde{q}_{(k, l)(j, i)}(x)(\tilde{f}(j, i)-\widetilde{f}(k, l)) \\
= & \sum_{j \in \mathbb{Z}_{+}}\left[q_{k j}(x)-q_{l j}(0)\right]^{+}(\widetilde{f}(j, l)-\widetilde{f}(k, l)) \\
& +\sum_{j \in \mathbb{Z}_{+}}\left[q_{l j}(0)-q_{k j}(x)\right]^{+}(\tilde{f}(k, j)-\widetilde{f}(k, l)) \\
& +\sum_{j \in \mathbb{Z}_{+}}\left[q_{k j}(x) \wedge q_{l j}(0)\right](\widetilde{f}(j, j)-\widetilde{f}(k, l)) .
\end{aligned}
$$

In what follows, we use the notation $\mathbb{E}_{x, i, j}$ and $\mathbb{P}_{x, i, j}$ to denote the corresponding conditional expectation and probability for the coupled process $(X(t), \alpha(t), \widehat{\alpha}(t))$ conditioned on $(X(0), \alpha(0), \widehat{\alpha}(0))=$ $(x, i, j)$. Let $\vartheta=\inf \{t \geq 0: \alpha(t) \neq \widehat{\alpha}(t)\}$. Define $\tilde{g}: \mathbb{Z} \times \mathbb{Z} \mapsto \mathbb{R}$ by $\widetilde{g}(k, l)=\mathbf{1}_{\{k=l\}}$. By the definition of the function $\widetilde{g}$, we have

$$
\begin{aligned}
\widetilde{Q}(x) \widetilde{g}(k, k) & =\sum_{j \in \mathbb{Z}_{+}, j \neq k}\left[q_{k j}(x)-q_{k j}(0)\right]^{+}+\sum_{j \in \mathbb{Z}_{+}, j \neq k}\left[q_{k j}(0)-q_{k j}(x)\right]^{+} \\
& =\sum_{j \in \mathbb{Z}_{+}, j \neq k}\left|q_{k j}(x)-q_{k j}(0)\right|=: \Xi(x, k) .
\end{aligned}
$$


For any $\varepsilon>0$, let $h>0$ such that $B_{h} \in D$ and $\sup _{(x, k) \in B_{h} \times \mathbb{Z}_{+}} \Xi(x, k)<\frac{\varepsilon}{2 T}$. Applying Itô's formula and noting that $\alpha(t)=\widehat{\alpha}(t), t \leq \vartheta$,

we obtain that

$$
\begin{aligned}
\mathbb{P}_{x, i, i}\left\{\vartheta \leq T \wedge \tau_{h}\right\} & =\mathbb{E}_{x, i, i} \widetilde{g}\left(\alpha\left(\vartheta \wedge T \wedge \tau_{h}\right), \widehat{\alpha}\left(\vartheta \wedge T \wedge \tau_{h}\right)\right) \\
& =\mathbb{E}_{x, i, i} \int_{0}^{\vartheta \wedge T \wedge \tau_{h}} \widetilde{Q}(X(t)) \widetilde{g}(\alpha(t), \widehat{\alpha}(t)) d t \\
& =\mathbb{E}_{x, i, i} \int_{0}^{\vartheta \wedge T \wedge \tau_{h}} \Xi(X(t), \alpha(t)) d t \\
& \leq T \sup _{(x, i) \in B_{h} \times \mathbb{Z}_{+}} \Xi(x, k) \leq \frac{\varepsilon}{2} .
\end{aligned}
$$

Thus In view of Lemma 3.1, there is $\delta>0$ such that $\mathbb{P}_{x, i, i}\left\{\tau_{h} \leq T\right\} \leq \frac{\varepsilon}{2}$. This and (A.6) derive

$$
\mathbb{P}_{x, i, i}\left\{\vartheta \wedge \tau_{h} \leq T\right\} \leq \mathbb{P}_{x, i, i}\left\{\vartheta \leq T \wedge \tau_{h}\right\}+\mathbb{P}_{x, i, i}\left\{\tau_{h} \leq T\right\} \leq \varepsilon
$$

We have that

$$
\begin{aligned}
\left|\mathbb{E}_{x, i} f(\alpha(t))-\mathbb{E}_{0, i} f(\alpha(t))\right| & =\left|\mathbb{E}_{x, i, i}[f(\alpha(t))-f(\widehat{\alpha}(t))]\right| \\
& =\left|\mathbb{E}_{x, i, i} \mathbf{1}_{\left\{\vartheta \wedge \tau_{h} \leq t\right\}}[f(\alpha(t))-f(\widehat{\alpha}(t))]\right| \\
& \leq 2 M_{f} \mathbb{P}_{x, i, i}\left\{\vartheta \wedge \tau_{h} \leq t\right\} \leq 2 M_{f} \varepsilon, \text { for } t \in[0, T] .
\end{aligned}
$$

where $M_{f}=\sup _{i \in \mathbb{Z}_{+}}|f(i)|$. The lemma is proved.

\section{References}

[1] W.J. Anderson, Continuous-time Markov chains: An Applications-Oriented Approach, Springer, 2012.

[2] L. Arnold, W. Kliemann, and E. Oeljeklaus. Lyapunov exponents of linear stochastic systems, Lyapunov exponents. Springer Berlin Heidelberg, 1986. 85-125.

[3] P. Baxendale, Invariant measures for nonlinear stochastic differential equations, Lyapunov exponents (Oberwolfach, 1990), Lecture Notes in Math., vol. 1486, Springer, Berlin, 1991, pp. 123140.

[4] M. Benaïm and C. Lobry, Lotka Volterra in fluctuating environment or "how switching between beneficial environments can make survival harder", Ann. Appl. Probab. (2016), to appear.

[5] M.F. Chen, From Markov Chains to Non-equilibrium Particle Systems, World Scientific, Singapore, 2004.

[6] R. Z. Khasminskii, Necessary and sufficient conditions for the asymptotic stability of linear stochastic systems, Theory Probab. Appl. 12.1 (1967): 144-147.

[7] R.Z. Khasminskii, C. Zhu, and G. Yin, Stability of regime-switching diffusions, Stochastic Process. Appl., 117 (2007), no. 8, 1037-1051.

[8] R. Liptser and A.N. Shiryayev, Theory of Martingales, (Vol. 49) Springer, 2012.

[9] X. Mao, Stability of stochastic differential equations with Markovian switching, Stochastic Process. Appl., 79 (1999),45-67.

[10] X. Mao, C. Yuan. Stochastic Differential Equations with Markovian Switching, Imperial College Press, London, 2006.

[11] D.H. Nguyen and G. Yin, Modeling and analysis of switching diffusion systems: past-dependent switching with a countable state space, SIAM J. Control Optim. 54 (2016), no. 5, 2450-2477. 
[12] D.H. Nguyen and G. Yin, Recurrence and Ergodicity of Switching Diffusions with PastDependent Switching Having A Countable State Space, submitted

[13] J. Shao and F. Xi, Stability and recurrence of regime-switching diffusion processes, SIAM J. Control Optim. 52 (2014), no. 6, 3496-3516.

[14] A.V. Skorokhod, Asymptotic Methods in the Theory of Stochastic Differential Equations, Vol. 78. American Mathematical Soc., 1989.

[15] F. Xi and G. Yin, Almost sure stability and instability for switching-jump-diffusion systems with state-dependent switching, J. Math. Anal. Appl. 400 (2013), no. 2, 460-474.

[16] F. Xi and L. Zhao, On the stability of diffusion processes with state-dependent switching, Sci. China, Ser. A, 49 (2006), 1258-1274

[17] F. Xi and C. Zhu, On Feller and Strong Feller Properties and Exponential Ergodicity of Regime-Switching Jump Diffusion Processes with Countable Regimes, submitted.

[18] G. Yin and F.B. Xi, Stability of regime-switching jump diffusions, SIAM J. Control Optim., 48 (2010), 4525-4549.

[19] G. Yin, B. Zhang, and C. Zhu, Practical stability and instability of regime-switching diffusions, J. Control Theory Appl. 6 (2008), no. 2, 105-114.

[20] G. Yin, G. Zhao, and F. Wu, Regularization and stabilization of randomly switching dynamic systems SIAM J. Appl. Math. 72 (2012), no. 5, 1361-1382.

[21] G. Yin and C. Zhu, Hybrid Switching Diffusions: Properties and Applications, Springer, New York, 2010.

[22] X. Zong, F. Wu, G. Yin, and Z. Jin, Almost Sure and $\mathrm{p}^{\text {th }}$-Moment Stability and Stabilization of Regime-Switching Jump Diffusion Systems, SIAM J. Control Optim. 52 (2014), no. 4, 25952622 . 